\title{
Wireless Powered Dense Cellular Networks: How Many Small Cells Do We Need?
}

\author{
Lifeng Wang, Member, IEEE, Kai-Kit Wong, Fellow, IEEE, \\ Robert W. Heath, Jr., Fellow, IEEE, and Jinhong Yuan, Fellow, IEEE
}

\begin{abstract}
This paper focuses on wireless powered 5G dense cellular networks, where base station (BS) delivers energy to user equipment (UE) via the microwave radiation in sub-6 GHz or millimeter wave (mmWave) frequency, and UE uses the harvested energy for uplink information transmission. By addressing the impacts of employing different numbers of antennas and bandwidths at lower and higher frequencies, we evaluate the amount of harvested energy and throughput in such networks. Based on the derived results, we obtain the required small cell density to achieve an expected level of harvested energy or throughput. Also, we obtain that when the ratio of the number of sub-6-GHz BSs to that of the mmWave BSs is lower than a given threshold, UE harvests more energy from an mmWave BS than a sub-6-GHz BS. We find how many mmWave small cells are needed to perform better than the sub-6-GHz small cells from the perspectives of harvested energy and throughput. Our results reveal that the amount of harvested energy from the mmWave tier can be comparable to the sub-6-GHz counterpart in the dense scenarios. For the same tier scale, mmWave tier can achieve higher throughput. Furthermore, the throughput gap between different mmWave frequencies increases with the mmWave BS density.
\end{abstract}

Index Terms-Millimeter wave (mmWave), dense small cells, wireless power transfer, throughput.

\section{INTRODUCTION}

W IRELESS power transfer (WPT) is an appealing approach to prolong the lifetime of user equipment (UE), when compared to the traditional energy harvesting sources such as solar and wind that highly depend upon the conditions of the environments. However, the implementation of WPT in conventional cellular networks may be challenging, due to the fact that it cannot support long-distance WPT in the absence of directed power beams, and small cells are not

Manuscript received November 12, 2016; revised March 15, 2017; accepted March 31, 2017. Date of publication June 28, 2017; date of current version August 18, 2017. This work was supported in part by the U.K. Engineering and Physical Sciences Research Council under Grant EP/M016005/1 and in part by the Australia Research Council Discovery Project under Grant DP160104566. This paper was presented at the IEEE International Conference on Communications, Paris, France, May 2017 [1]. (Corresponding author: Lifeng Wang.)

L. Wang and K.-K. Wong are with the Department of Electronic and Electrical Engineering, University College London, London WC1E 7JE, U.K. (e-mail: lifeng.wang@ucl.ac.uk; kai-kit.wong@ucl.ac.uk).

R. W. Heath, Jr., is with the Department of Electrical and Computer Engineering, The University of Texas at Austin, Austin, TX 78712 USA (e-mail: rheath@ece.utexas.edu).

J. Yuan is with the School of Electrical Engineering and Telecommunications, University of New South Wales, Sydney, NSW 2052, Australia (e-mail: j.yuan@unsw.edu.au).

Color versions of one or more of the figures in this paper are available online at http://ieeexplore.iee.org.

Digital Object Identifier 10.1109/JSAC.2017.2720858 densely deployed [2]. In the fifth generation (5G) cellular networks, technologies such as millimeter wave (mmWave), massive multiple-input multiple-output (MIMO) and ultradense small cells in the sub- $6 \mathrm{GHz}$ and mmWave frequencies will be adopted [3]-[5], which make next-generation networks more suitable for WPT, due to at least the following two key factors:

- The very sharp signal beams in large-scale antenna systems such as massive MIMO and mmWave bring large antenna array gains, enabling WPT over long distances.

- Dense small cells will be deployed to provide proximity services, which drastically reduce propagation loss for WPT. In 5G ultra-dense networks (UDNs) [6], the distance between a UE and its serving base station (BS) will be much shorter than ever before.

Therefore, $5 \mathrm{G}$ networks provide a wealth of opportunities for WPT.

In this paper, we study wireless powered dense cellular networks, in which active UE may select a sub-6 GHz or mmWave BS as dedicated RF energy source, and utilizes its harvested energy for uplink information transmission. We provide a tractable analytical framework to characterize both the energy harvesting and throughput performance in such networks. This work will answer how many sub- $6 \mathrm{GHz} / \mathrm{mmWave}$ small cells are needed in order to achieve some target harvested energy and throughput. In particular, we derive the number of mmWave small cells that is required to achieve better performance than the sub-6 GHz counterpart.

\section{A. Prior Work and Motivation}

Early works have studied the potential of wireless energy harvesting in traditional systems. In [7], simultaneous wireless information and power transfer (SWIPT) was investigated in a MIMO wireless broadcasting channel, and two RF energy harvesting designs at the receiver were investigated, namely time switching and power splitting. In [8], each single-antenna UE was considered to harvest ambient RF energy from the surrounding single-antenna access points in a wireless powered network, and a spatial throughput maximization problem was formulated. The work of [9] then studied wireless energy harvesting in the sensor networks, where many battery-free sensors are powered by a number of ambient RF energy sources. The power allocation problem in a wireless energy harvesting enabled relay network was considered in [10], where the energy constrained relay used power splitting for cooperative transmission. In [11], $K$-tier uplink cellular 
networks with ambient RF energy harvesting were considered, and the uplink coverage probability was derived. In device-to-device (D2D) underlaying homogeneous cellular networks, [12] investigated wireless energy harvesting enabled D2D transmissions under different spectrum access policies. Most recently in heterogeneous cellular network $(\mathrm{HCN})$ with energy harvesting based D2Ds, [13] studied the D2D relaying in D2D communications. However, the prior work [7]-[13] only addresses the effects of current network features on WPT. Therefore, new research on WPT under emerging $5 \mathrm{G}$ network architectures is needed.

The rapid development of $5 \mathrm{G}$ technologies has encouraged more research on WPT. In [14], the optimal power transfer beamforming was asymptotically derived by considering large number of antennas in a single massive MIMO cell, and the optimal solution for maximizing the throughput under the user fairness criterion was also asymptotically obtained. The work of [15] provided an overview of SWIPT in massive distributed antenna systems. In [16], WPT was applied to recharge UEs in massive MIMO aided $K$-tier HCNs, where UEs with large energy storage are connected to their BSs based on two typical user association schemes. Although the work in [14]-[16] has studied the impact of massive MIMO antennas on WPT, they only focused on the sub-6 GHz networks. Moreover, [14] and [15] only considered a single massive MIMO cell case, and [16] did not study the more practical case of UEs with finite battery capacity.

Existing work has studied the coverage and capacity in the mmWave cellular networks without WPT based on field measurements [17] or stochastic models [18]-[20], where constant transmit power was assumed. However, in the wireless powered mmWave networks, coverage and capacity need to be re-studied, since UE's transmit power becomes random and depends on the harvested energy. The use of mmWave for WPT is promising because of the fact that directed beams are used in mmWave communications and mmWave small cells will also be more densely deployed. Recent efforts on WPT have thus turned to the use of mmWave bands. In particular, the hardware design of the mmWave rectifier circuit for WPT has been studied in, e.g., [21] and [22]. The work of [23] studied the wireless powered mmWave cellular network, in which uniform linear array with analog beamforming was implemented for WPT and uplink information transmission. Subsequently in [24], downlink SWIPT was investigated in mmWave systems, and the average harvested energy at the UEs and the downlink coverage probability were evaluated. However, the limitation of [23] is that it assumed that mmWave UEs have infinite battery capacity such that constant uplink transmit power can be guaranteed, while [24] only considered WPT in the downlink and investigated the effects of WPT on downlink information transmission. To the best of our knowledge, wireless powered 5G with both sub- $6 \mathrm{GHz}$ and mmWave frequency bands is an open area of research.

In wireless powered cellular networks encompassing sub-6 GHz BSs and mmWave BSs equipped with their respective antenna arrays, WPT can operate at different frequencies, and a UE with finite battery capacity may harvest RF energy in the sub-6 GHz tier or the mmWave tier for accomplishing uplink information transmission. Nevertheless, such networks are not well understood. Moreover, under 5G realistic settings, how many small cells need to be deployed for supporting WPT and information transmission is still unknown.

\section{B. Contributions and Organization}

In this paper, we study wireless powered $5 \mathrm{G}$ dense cellular networks, in which sub-6 GHz or mmWave BSs can be selected to power UEs with finite battery capacity. Our analysis permits to account for the key characteristics of sub- $6 \mathrm{GHz}$ and mmWave channels and the effects of different antenna array gains and node densities. In summary, we have made the following major contributions:

- We model a wireless powered cellular network consisting of sub-6 GHz BSs and mmWave BSs equipped with antenna arrays, with the help of stochastic geometry. In the energy harvesting phase, each sub- $6 \mathrm{GHz}$ BS delivers energy to the nearest sub-6 GHz UE using maximum-ratio transmission (MRT) beamforming, and each mmWave BS delivers mmWave RF energy to the mmWave UE that has the minimum pathloss via narrow beam. In the uplink transmission phase, each active UE uses the harvested energy to transmit information to its associated BS.

- We derive the energy coverage probability in sub-6 GHz and mmWave tiers by considering both the directed transferred power from the associated BS and the ambient RF energy from nearby BSs. We find that when the sub-6 GHz small cell density is lower than a given threshold, a UE harvests more RF energy from a mmWave BS than a sub-6 GHz BS. By considering WPT mode selection, we further derive the probability that a UE selects a sub- $6 \mathrm{GHz} \mathrm{BS}$, line-ofsight $(\mathrm{LoS})$ mmWave BS or a non-LoS (NLoS) mmWave BS for WPT.

- Also, we derive the throughput in the uplink sub- $6 \mathrm{GHz}$ and mmWave tiers with different bandwidths. Based on the results, the number of sub- $6 \mathrm{GHz} / \mathrm{mm}$ Wave small cells that are required to achieve a targeted throughput is obtained. We demonstrate that the ratio $\kappa_{\mu} \mathrm{UE}$ of sub-6 GHz BS density to active sub-6 GHz UE density should be greater than a certain threshold, in order to obtain the desired performance. The throughput grows at a higher speed when increasing $\kappa_{\mu \mathrm{UE}}$, compared to increasing the number of BS antennas. Moreover, we calculate how many mmWave small cells are needed such that the achievable throughput in the mmWave tier is larger than that in the sub- $6 \mathrm{GHz}$ tier.

- Simulation results have confirmed our analysis, and illustrated that the amount of harvested energy is dominated by directed power transfer, compared to the ambient RF energy harvesting. The amount of harvested energy from ambient mmWave RF can still be larger than the sub- $6 \mathrm{GHz}$ counterpart in the ultra-dense mmWave tier. When power transfer mode selection is supported, the probability that a UE selects NLoS BS for WPT is negligible, and LoS mmWave WPT is also comparable 
to the sub-6 GHz counterpart in terms of energy coverage. It is revealed that in the dense scenario where each tier has the same number of BSs and active UEs, a mmWave UE can achieve a higher throughput than the sub- $6 \mathrm{GHz}$ counterpart. Furthermore, the performance gap between different mmWave frequencies increases with mmWave BS density due to the fact that more densification gains can be obtained at lower mmWave frequencies.

The remainder of the paper is organized as follows. Section II describes the system model including the energy harvesting and information transmission. Section III and Section IV analyze the energy harvesting and throughput in the considered networks, respectively. After that, we present our simulation results in Section V. Finally, Section VI gives conclusions.

Notations: $(\cdot)^{H}$ denotes the conjugate transpose operator; $\mathcal{C N}(\mathbf{0}, \Lambda)$ represents the complex Gaussian distribution with zero mean and covariance matrix $\Lambda ;\|\cdot\|$ is the Euclidean norm; $\mathbb{E}[\cdot]$ denotes the expectation operator; $\mathbf{0}_{M \times N}$ is the $M \times N$ zero matrix, and $\mathbf{I}_{M}$ is the $M \times M$ identity matrix.

\section{SYSTEM DESCRIPTIONS}

\section{A. Network Model}

We consider a wireless powered cellular network consisting of the sub- $6 \mathrm{GHz}$ and mmWave small cells, ${ }^{1}$ where UEs are powered by the RF energy from the BSs before uplink communication. Each sub- $6 \mathrm{GHz}$ BS has an array of $N$ sub$6 \mathrm{GHz}$ antennas, and each mmWave BS is equipped with a large mmWave antenna array. Each sub-6 GHz UE $(\mu \mathrm{UE})$ is equipped with a single sub-6 GHz antenna, while each mmWave UE (mmUE) is equipped with a small mmWave antenna array, since it is expected that the shorter mmWave wavelengths would enable UEs to fit more antennas for a fixed antenna aperture. The sub- $6 \mathrm{GHz}$ BSs are randomly located following a homogeneous Poisson point process (HPPP) $\Phi_{\mu}\left(\lambda_{\mu}\right)$ with the density $\lambda_{\mu}$, and the mmWave BSs are randomly located following an independent HPPP $\Phi_{\mathrm{mm}}\left(\lambda_{\mathrm{mm}}\right)$ with the density $\lambda_{\mathrm{mm}}$.

When a UE requires the directed power transfer from a dedicated $\mathrm{BS}$, a $\mu \mathrm{UE}$ will be connected to the sub- $6 \mathrm{GHz}$ BS that provides the largest received sub- $6 \mathrm{GHz}$ signal power, and accordingly, a mmUE will be connected to the mmWave BS that provides the largest received mmWave signal power.

We assume that all sub-6 GHz channels are subject to independent identically distributed (IID) quasi-static Rayleigh block fading, which matches well with practical NLoS measurements [25], [26]. In addition, for large number of antennas, the effect of small-scale fading is considered averaged out [25], [27] and the sub-6 $\mathrm{GHz}$ channel power gain is dominated by random pathloss. As a consequence,

\footnotetext{
${ }^{1}$ In the future wireless networks such as $5 \mathrm{G}$, both sub- $6 \mathrm{GHz}$ and mmWave frequency bands will be applied [3]. In such networks, sub-6 GHz BSs and mmWave BSs equipped with different antenna arrays coexist, which serve UEs that operate on the sub-6 GHz or mmWave frequency bands, respectively.
}

Rayleigh channel distribution is suitable for modeling sub-6 GHz links when the number of antennas grows large, and has also been used in the literature such as [25] and [26] for studying $5 \mathrm{G}$ sub-6 $\mathrm{GHz}$ scenarios. In the mmWave systems, the high free-space mmWave pathloss leads to very limited spatial selectivity or scattering, and thus the traditional small-scale fading distributions are invalid for modeling the sparse scatting mmWave environments [28]. As suggested in the channel measurement work [17], the effect of small-scale fading in mmWave channels is omitted in this paper by considering highly directional transmissions. ${ }^{2}$

\section{B. Energy Harvesting}

1) Sub-6 GHz Tier: In the sub-6 GHz tier, each sub-6 GHz BS adopts MRT beamforming to transfer the energy for recharging its $\mu \mathrm{UE}$, to maximize the transferred power. Thanks to the high diffraction and penetration characteristics of sub- $6 \mathrm{GHz}$ signals, the blockage effect in the sub- $6 \mathrm{GHz}$ channel is less significant than the mmWave counterpart [26], [29]. To simplify our analysis, shadow fading [30] is omitted in the sub- $6 \mathrm{GHz}$ tier of this paper, which is commonly-seen in the literature such as [20], [24], and [26] for tractability. Hence, for a typical $\mu \mathrm{UE}$, say $o$, connected with its serving sub-6 GHz BS, its instantaneous harvested power is written as

$$
\begin{aligned}
& P_{r}^{\mu}=\underbrace{\eta_{\mu} P_{\mu}\left\|\mathbf{h}_{o}\right\|^{2} L\left(\left|X_{o}\right|\right)}_{\Lambda_{\mu_{1}}} \\
&+\underbrace{\eta_{\mu} \sum_{k \in \Phi_{\mu}\left(\lambda_{\mu}\right) \backslash\{o\}} P_{\mu}\left|\mathbf{h}_{k, o} \frac{\mathbf{h}_{k}^{H}}{\left\|\mathbf{h}_{k}\right\|}\right|^{2} L\left(\left|X_{k, \mu}\right|\right)}_{\Lambda_{\mu_{2}}},
\end{aligned}
$$

where $\Lambda_{\mu_{1}}$ is the directed transferred power, and $\Lambda_{\mu_{2}}$ is the total power from the ambient sub- $6 \mathrm{GHz} \mathrm{RF}, \eta_{\mu}$ is the sub-6 GHz RF-to-DC conversion efficiency, $P_{\mu}$ is the transmit power of sub-6 GHz BS, $\mathbf{h}_{o} \sim \mathcal{C N}\left(\mathbf{0}, \mathbf{I}_{N}\right)$ and $\left|X_{o}\right|$ are the small-scale fading channel vector and distance between the typical $\mu \mathrm{UE}$ and its serving BS, respectively, $L\left(\left|X_{o}\right|\right)=$ $\beta_{\mu}\left(\left|X_{o}\right|\right)^{-\alpha_{\mu}}$ is the pathloss function with the exponent $\alpha_{\mu}$, where $\beta_{\mu}$ is a frequency dependent constant value, which is commonly set as $\left(\frac{c}{4 \pi f_{c}}\right)^{2}$ with $c=3 \times 10^{8} \mathrm{~m} / \mathrm{s}$ and the carrier frequency $f_{c}, \frac{\mathbf{h}_{k}^{H}}{\left\|\mathbf{h}_{k}\right\|}$ is the MRT beamforming vector of the sub-6 GHz BS $k\left(k \in \Phi_{\mu} \backslash\{o\}\right)$ with $\mathbf{h}_{k} \sim \mathcal{C N}\left(\mathbf{0}, \mathbf{I}_{N}\right)$, $\mathbf{h}_{k, o} \sim \mathcal{C N}\left(\mathbf{0}, \mathbf{I}_{N}\right)$ and $\left|X_{k, \mu}\right|$ are the small-scale fading channel vector and the distance between the typical $\mu \mathrm{UE}$ and the sub-6 GHz BS $k$ (except the serving sub-6 GHz BS), respectively.

2) mmWave Tier: In the mmWave tier, a sectored model is applied to analyze the beam pattern [19], [20], [31], i.e., the effective antenna gain for a mmWave BS $\ell\left(\ell \in \Phi_{\mathrm{mm}} \backslash\{o\}\right)$

\footnotetext{
${ }^{2}$ Note that in some existing work such as [19] and [20], it has been mentioned that when assuming that mmWave channel undergoes Rayleigh fading [20] or Nakagami fading [19], the tractability of analysis can be improved.
} 
seen by the typical mmUE $o$ is expressed as

$$
G_{\ell}= \begin{cases}\mathrm{M}_{\mathrm{B}} \mathrm{M}_{\mathrm{D}}, & \operatorname{Pr}_{\mathrm{M}_{\mathrm{B}} \mathrm{M}_{\mathrm{D}}}=\frac{\theta_{\mathrm{B}} \theta_{\mathrm{D}}}{4 \pi^{2}}, \\ \mathrm{M}_{\mathrm{B}} \mathrm{m}_{\mathrm{D}}, & \operatorname{Pr}_{\mathrm{M}_{\mathrm{B}} \mathrm{m}_{\mathrm{D}}}=\frac{\theta_{\mathrm{B}}\left(2 \pi-\theta_{\mathrm{D}}\right)}{4 \pi^{2}}, \\ \mathrm{~m}_{\mathrm{B}} \mathrm{M}_{\mathrm{D}}, & \operatorname{Pr}_{\mathrm{m}_{\mathrm{B}} \mathrm{M}_{\mathrm{D}}=\frac{\left(2 \pi-\theta_{\mathrm{B}}\right) \theta_{\mathrm{D}}}{4 \pi^{2}},} \\ \mathrm{~m}_{\mathrm{B}} \mathrm{m}_{\mathrm{D}}, & \operatorname{Pr}_{\mathrm{m}_{\mathrm{B}} \mathrm{m}_{\mathrm{D}}}=\frac{\left(2 \pi-\theta_{\mathrm{B}}\right)\left(2 \pi-\theta_{\mathrm{D}}\right)}{4 \pi^{2}},\end{cases}
$$

where $\mathrm{M}_{\mathrm{B}}, \mathrm{m}_{\mathrm{B}}$, and $\theta_{\mathrm{B}}$ are the main lobe gain, side lobe gain, and half power beamwidth of the mmWave BS antenna, respectively, and $M_{D}, m_{D}$, and $\theta_{D}$ are the main lobe gain, side lobe gain, and half power beamwidth of the mmUE antenna, respectively. We assume that the maximum array gain $\mathrm{M}_{\mathrm{B}} \mathrm{M}_{\mathrm{D}}$ can be obtained for the typical BS and its mmUE.

Recognizing that mmWave communication is sensitive to the blockage in the outdoor scenario, a mmUE is associated with either a LoS mmWave BS or a NLoS mmWave BS. We denote $f_{\operatorname{Pr}}(R)$ as the probability that a link at a distance $R$ is LoS, so that the NLoS probability of a link is $1-f_{\operatorname{Pr}}(R)$. We consider two different pathloss laws: $L(R)=$ $\beta_{\mathrm{LoS}}^{\mathrm{mm}} R^{-\alpha_{\mathrm{LOS}}^{\mathrm{mm}}}$ is the pathloss function for LoS channel and $L(R)=\beta_{\mathrm{NLoS}}^{\mathrm{mm}} R^{-\alpha_{\mathrm{NLoS}}^{\mathrm{mm}}}$ is the pathloss function for NLoS channel, where $\beta_{\mathrm{LoS}}^{\mathrm{mm}}, \beta_{\mathrm{NLoS}}^{\mathrm{mm}}$ are the frequency dependent constant values and $\alpha_{\mathrm{LoS}}^{\mathrm{mm}}, \alpha_{\mathrm{NLoS}}^{\mathrm{mm}}$ are the pathloss exponents.

For a typical mmUE $o$ connected with its serving mmWave BS, its instantaneous harvested power is written as

$$
\begin{aligned}
P_{r}^{\mathrm{mm}}=\underbrace{\eta_{\mathrm{mm}} P_{\mathrm{mm}} \mathrm{M}_{\mathrm{B}} \mathrm{M}_{\mathrm{D}} L\left(\left|Y_{o}\right|\right)}_{\Lambda_{\mathrm{mm}_{1}}} & \underbrace{\eta_{\mathrm{mm}} \sum_{\ell \in \Phi_{\mathrm{mm}}\left(\lambda_{\mathrm{mm}}\right) \backslash\{o\}} P_{\mathrm{mm}} G_{\ell} L\left(\left|Y_{\ell, \mathrm{mm}}\right|\right)}_{\Lambda_{\mathrm{mm}_{2}}},
\end{aligned}
$$

where $\Lambda_{\mathrm{mm}_{1}}$ is the directed transferred power, and $\Lambda_{\mathrm{mm}}$ is the total power from the ambient mmWave RF, $\eta_{\mathrm{mm}}$ is the mmWave RF-to-DC conversion efficiency, $P_{\mathrm{mm}}$ is the mmWave BS transmit power, $\left|Y_{o}\right|$ is the distance between the typical mmUE and its serving mmWave $\mathrm{BS}$, and $\left|Y_{\ell, \mathrm{mm}}\right|$ is the distance between the typical mmUE and the mmWave BS $\ell \in \Phi_{\mathrm{mm}} \backslash\{o\}$ (except the serving mmWave BS).

\section{Uplink Transmission}

1) Sub-6 GHz Tier: In the sub-6 GHz tier, maximum-ratio combining (MRC) is utilized for maximizing the received signal power at the sub- $6 \mathrm{GHz} \mathrm{BS}$. The receive signal-tointerference-plus-noise ratio (SINR) of a typical sub-6 GHz $\mathrm{BS}$ from its intended $\mu \mathrm{UE}$ is therefore given by

$$
\operatorname{SINR}_{\mu}=\frac{S_{\left(\lambda_{\mu}\right)}^{\mu}}{I_{\left(\bar{\lambda}_{\mu \mathrm{UE}}\right)}^{\mu}+\sigma^{2}}
$$

where

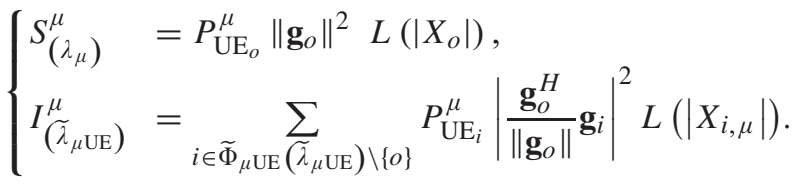

In (5), $P_{\mathrm{UE}_{i}}^{\mu}$ denotes the $i$-th $\mu \mathrm{UE}$ 's transmit power, $\mathbf{g}_{o} \sim$ $\mathcal{C N}\left(\mathbf{0}, \mathbf{I}_{N}\right)$ is the small-scale fading channel vector between the typical sub- $6 \mathrm{GHz}$ BS and its intended $\mu \mathrm{UE}, \widetilde{\Phi}_{\mu \mathrm{UE}}\left(\widetilde{\lambda}_{\mu \mathrm{UE}}\right)$ is the point process for the active $\mu \mathrm{UEs}$ with density $\widetilde{\lambda}_{\mu \mathrm{UE}}$, $\mathbf{g}_{i} \sim \mathcal{C N}\left(\mathbf{0}, \mathbf{I}_{N}\right)$ and $\left|X_{i, \mu}\right|$ are the small-scale fading channel vector and the distance between the typical sub- $6 \mathrm{GHz}$ BS and interfering $\mu \mathrm{UE} i$, respectively, and $\sigma^{2}$ is the noise power.

2) mmWave Tier: In the mmWave tier, we only consider the LoS uplink transmissions, since each mmUE uses lower transmit power from limited harvested energy and the harvested energy from NLoS link is much lower, which means that NLoS uplink will be blocked. According to the LoS mmWave model in [19] and [26], the receive SINR of a typical mmWave BS from its intended mmUE is given by

$$
\mathrm{SINR}_{\mathrm{mm}}=\frac{S_{\left(\lambda_{\mathrm{mm}}\right)}^{\mathrm{mm}}}{I_{\left(\bar{\lambda}_{\mathrm{mmUE}}\right)}^{\mathrm{mm}}+\sigma^{2}}
$$

where

$$
\left\{\begin{array}{l}
S_{\left(\lambda_{\mathrm{mm}}\right)}^{\mathrm{mm}}=P_{\mathrm{UE}_{o}}^{\mathrm{mm}} \mathrm{M}_{D} \mathrm{M}_{B} L\left(\left|Y_{o}\right|\right) \mathbf{1}\left(\left|Y_{o}\right|<R_{\mathrm{LoS}}\right) \\
I_{\left.\widetilde{\lambda}_{\mathrm{mmUE}}\right)}^{\mathrm{mm}}=\sum_{\left.j \in \widetilde{\Phi}_{\mathrm{mmUE}} \widetilde{\lambda}_{\mathrm{mmUE}}\right) \backslash\{o\}} P_{\mathrm{UE}_{j}}^{\mathrm{mm}} \widetilde{G}_{j} L_{\mathbf{1}}\left(\left|Y_{j, \mu}\right|\right)
\end{array}\right.
$$

with $L_{\mathbf{1}}\left(\left|Y_{j, \mu}\right|\right)=L\left(\left|Y_{j, \mu}\right|\right) \mathbf{1}\left(\left|Y_{j, \mu}\right|<R_{\mathrm{LoS}}\right)$. Here, $P_{\mathrm{UE}_{o}}^{m m}$ is the typical mmUE's transmit power and $P_{\mathrm{UE}_{j}}^{\mathrm{mm}}$ is the $j$-th interfering mmUE's transmit power, $1(\cdot)$ is the indicator function that returns one if the condition is satisfied and zero otherwise, $R_{\mathrm{LoS}}$ denotes the maximum distance that LoS can be guaranteed [19], [26] (i.e., $f_{\operatorname{Pr}}(R)=1$ as $R \leq R_{\mathrm{LoS}}$ and otherwise $f_{\mathrm{Pr}}(R)=0$.), $\widetilde{\Phi}_{\text {mmUE }}\left(\widetilde{\lambda}_{\mathrm{mmUE}}\right)$ is the point process corresponding to the active mmUEs with the density $\widetilde{\lambda}_{\text {mmUE }}, \widetilde{G}_{j}$ is the effective antenna gain for an interfering mmUE $j$ seen by the typical mmWave BS, which follows the distribution given in (2), and $\left|Y_{j, \mu}\right|$ is the distance between the interfering mmUE $j$ and the typical mmWave BS.

\section{ENERGY HARVESTING}

We evaluate the wireless energy harvesting in the sub-6 GHz and mmWave cellular networks. To gain comprehensive understanding, we respectively examine the directed transferred power from the associated BS and the ambient RF harvested power from nearby BSs that a UE can obtain, which allows us to quantify the harvested energy from the dedicated RF and ambient RF. Note that the minimum amount of energy is required to activate the harvesting circuit, which depends on the specific circuit designs based on CMOS, HSMS, SMS and etc. As surveyed in [32], the minimum RF input power for CMOS technology can be as low as $-27 \mathrm{dBm}$ based on the prior circuit work from 2006 to 2014. Therefore, the RF energy harvesting sensitivity level is much lower and can be omitted [8], [33]. In fact, since we consider 5G dense cellular networks, where dense BSs act as dedicated RF energy sources to power UEs via narrow beams, the amount of the received energy at a UE will be much larger than the RF energy harvesting sensitivity level [32]. 


\section{A. Directed Transferred Power}

1) Sub-6 GHz Tier: In the sub-6 GHz tier, given a power threshold $P_{\text {th }}$, the coverage probability that the directed transferred power is larger than $P_{\text {th }}$ can be derived as

$$
\begin{aligned}
& \Psi_{\mathrm{D}}^{\mu}\left(P_{\mathrm{th}}\right)= \sum_{n=0}^{N-1}\left(\frac{P_{\mathrm{th}}}{\eta_{\mu} P_{\mu} \beta_{\mu}}\right)^{n} \frac{2 \pi \lambda_{\mu}}{n !} \\
& \times \int_{0}^{\infty} e^{-\frac{r^{\alpha \mu} P_{\mathrm{th}}}{\eta_{\mu} P_{\mu} \beta_{\mu}}-\pi \lambda_{\mu} r^{2}} r^{\alpha_{\mu} n+1} d r .
\end{aligned}
$$

Proof: Based on (1), $\bar{\Psi}_{\mathrm{D}}^{\mu}\left(P_{\mathrm{th}}\right)$ is calculated as

$$
\begin{aligned}
\bar{\Psi}_{\mathrm{D}}^{\mu}\left(P_{\mathrm{th}}\right) & =\operatorname{Pr}\left(\Lambda_{\mu_{1}}>P_{\mathrm{th}}\right) \\
& =\int_{0}^{\infty} \operatorname{Pr}\left(\eta_{\mu} P_{\mu}\left\|\mathbf{h}_{o}\right\|^{2} \beta_{\mu} r^{-\alpha_{\mu}}>P_{\mathrm{th}}\right) f_{\left|X_{o}\right|}(r) d r
\end{aligned}
$$

where $f_{\left|X_{o}\right|}(r)$ is the probability density function (PDF) of the distance between a sub- $6 \mathrm{GHz}$ UE and its nearest sub- $6 \mathrm{GHz}$ $\mathrm{BS}$, which is given by

$$
f_{\left|X_{o}\right|}(r)=2 \pi \lambda_{\mu} r \exp \left(-\pi \lambda_{\mu} r^{2}\right) .
$$

Considering that $\left\|\mathbf{h}_{o}\right\|^{2} \sim \Gamma(N, 1)$, we can further calculate (9) as

$$
\bar{\Psi}_{\mathrm{D}}^{\mu}\left(P_{\mathrm{th}}\right)=\sum_{n=0}^{N-1} \int_{0}^{\infty} \frac{e^{-\frac{r^{\alpha} \mu P_{\mathrm{th}}}{\eta_{\mu} P_{\mu} \beta_{\mu}}}}{n !}\left(\frac{r^{\alpha} \mu P_{\mathrm{th}}}{\eta_{\mu} P_{\mu} \beta_{\mu}}\right)^{n} f_{\left|X_{o}\right|}(r) d r .
$$

Substituting (10) into (11), we obtain (8).

Based on (8), the sufficient condition for $\Psi_{\mathrm{D}}^{\mu}\left(P_{\mathrm{th}}\right)>\varepsilon$ $(0<\varepsilon<1)$ for a given $P_{\text {th }}$, is given by the following corollary.

Corollary 1: The probability of the achievable directed transferred power $P_{\text {th }}$ is larger than $\varepsilon$, if the sub-6 GHz BS density satisfies

$$
\lambda_{\mu}>\omega_{o}\left(\frac{\eta_{\mu} P_{\mu} \beta_{\mu} N}{P_{\mathrm{th}}}\right)^{-2 / \alpha_{\mu}}
$$

with large $N$, where $\omega_{o}=\ln \left(\frac{1}{1-\varepsilon}\right) / \pi$.

Proof: With large $N,\left\|\mathbf{h}_{o}\right\|^{2} \approx N$ [34], and (9) can be approximated as

$$
\begin{aligned}
\widetilde{\Psi}_{\mathrm{D}}^{\mu}\left(P_{\mathrm{th}}\right) & =\int_{0}^{\left(\frac{\eta_{\mu} P_{\mu} \beta_{\mu} N}{P_{\mathrm{th}}}\right)^{1 / \alpha_{\mu}}} f_{\left|X_{o}\right|}(r) d r \\
& =1-\exp \left(-\pi \lambda_{\mu}\left(\frac{\eta_{\mu} P_{\mu} \beta_{\mu} N}{P_{\mathrm{th}}}\right)^{2 / \alpha_{\mu}}\right) .
\end{aligned}
$$

Letting $\widetilde{\Psi}_{\mathrm{D}}^{\mu}\left(P_{\text {th }}\right)>\varepsilon$, after manipulations, gives (12).

It is indicated from Corollary 1 that the number of sub-6 GHz BSs needs to be large enough for WPT. Moreover, the required BS density decreases when adding more BS antennas, due to the fact that the decreased densification gains can be redeemed by obtaining more antenna gains.
2) mmWave Tier: In the mmWave tier, the coverage probability that the directed transferred power is larger than a threshold $P_{\text {th }}$ can be derived as

$$
\begin{aligned}
\Psi_{\mathrm{D}}^{\mathrm{mm}}\left(P_{\mathrm{th}}\right)=2 \pi \lambda_{\mathrm{mm}}[ & \int_{0}^{\varsigma\left(\beta_{\mathrm{LoS}}^{\mathrm{mm}}, \alpha_{\mathrm{LoS}}^{\mathrm{mm}}\right)} y f_{\mathrm{Pr}}(y) \\
& \times e^{-2 \pi \lambda_{\mathrm{mm}}\left[\Theta(y)+\Xi\left(\Im_{\mathrm{LoS}}\right)\right]} d y \\
& +\int_{0}^{\varsigma\left(\beta_{\mathrm{NLOS}}^{\mathrm{mm}}, \alpha_{\mathrm{NLoS}}^{\mathrm{mm}}\right)} y\left(1-f_{\mathrm{Pr}}(y)\right) \\
& \left.\times e^{-2 \pi \lambda_{\mathrm{mm}}\left[\Theta\left(\Im_{\mathrm{NLoS}}\right)+\Xi(y)\right]} d y\right]
\end{aligned}
$$

where $\varsigma\left(y_{1}, y_{2}\right)=\left(\frac{\eta_{\mathrm{mm}} P_{\mathrm{mm}} \mathrm{M}_{\mathrm{B}} \mathrm{M}_{\mathrm{D}}}{P_{\mathrm{th}}} y_{1}\right)^{1 / y_{2}}, \Theta(y)=\int_{0}^{y} t f_{\mathrm{Pr}}(t) d t$, $\Xi(y)=\int_{0}^{y}\left(1-f_{\operatorname{Pr}}(t)\right) t d t, \quad \Im_{\mathrm{LoS}}=\left(\frac{\beta_{\mathrm{NLoS}}^{\mathrm{mm}}}{\beta_{\mathrm{LoS}}^{\mathrm{mm}}}\right)^{1 / \alpha_{\mathrm{NLoS}}^{\mathrm{mm}}}$ $y^{\alpha_{\mathrm{LoS}}^{\mathrm{mm}} / \alpha_{\mathrm{NLOS}}^{\mathrm{mm}}}$, and $\Im_{\mathrm{NLoS}}=\left(\frac{\beta_{\mathrm{LOS}}^{\mathrm{mm}}}{\beta_{\mathrm{NLoS}}^{\mathrm{IIII}}}\right)^{1 / \alpha_{\mathrm{LoS}}^{\mathrm{mm}}} y^{\alpha_{\mathrm{NLOS}}^{\mathrm{mm}} / \alpha_{\mathrm{LoS}}^{\mathrm{mm}}}$.

Proof: Based on (3), $\bar{\Psi}_{\mathrm{D}}^{\mathrm{mm}}\left(P_{\mathrm{th}}\right)$ is calculated as

$$
\begin{aligned}
& \bar{\Psi}_{\mathrm{D}}^{\mathrm{mm}}\left(P_{\mathrm{th}}\right)=\operatorname{Pr}\left(\Lambda_{\mathrm{mm}_{1}}>P_{\mathrm{th}}\right) \\
& =\Upsilon_{\mathrm{LoS}} \int_{0}^{\varsigma\left(\beta_{\mathrm{LoS}}^{\mathrm{mm}}, \alpha_{\mathrm{LoS}}^{\mathrm{mm}}\right)} f_{R}^{\mathrm{LoS}}(y) d y \\
& +\Upsilon_{\mathrm{NLOS}} \int_{0}^{\varsigma\left(\beta_{\mathrm{NLoS}}^{\mathrm{mm}}, \alpha_{\mathrm{NLOS}}^{\mathrm{mm}}\right)} f_{R}^{\mathrm{NLoS}}(y) d y,
\end{aligned}
$$

where $\Upsilon_{\text {LoS }}$ represents the probability that the typical user is connected to a LoS BS and $\Upsilon_{\mathrm{NLOS}}=1-\Upsilon_{\mathrm{LoS}}$ represents the probability that the typical user is connected to a NLoS BS, $f_{R}^{\mathrm{LoS}}(x)$ is the conditioned PDF of the distance between the typical mmUE and its serving $\operatorname{LoS} \mathrm{BS}$, and $f_{R}^{\mathrm{NLoS}}(x)$ is the conditioned PDF of the distance between the typical mmUE and its serving NLoS BS, which are given by [19]

$$
f_{R}^{\mathrm{LoS}}(y)=\frac{2 \pi \lambda_{\mathrm{mm}}}{\Upsilon_{\mathrm{LoS}}} y f_{\mathrm{Pr}}(y) e^{-2 \pi \lambda_{\mathrm{mm}}\left[\Theta(y)+\Xi\left(\Im_{\mathrm{LoS}}\right)\right]},
$$

and

$f_{R}^{\mathrm{NLoS}}(y)=\frac{2 \pi \lambda_{\mathrm{mm}}}{\Upsilon_{\mathrm{NLoS}}} y\left(1-f_{\mathrm{Pr}}(y)\right) e^{-2 \pi \lambda_{\mathrm{mm}}\left[\Theta\left(\Im_{\mathrm{NLoS}}\right)+\Xi(y)\right]}$,

respectively. Substituting (16) and (17) into (15) yields (14).

Corollary 2: Given a threshold $P_{\text {th }}$, the coverage probability of the directed power transfer in the mmWave tier is larger than that in the sub- $6 \mathrm{GHz}$ tier under the following condition:

$$
\lambda_{\mu}<\frac{\ln \left(1-\bar{\Psi}_{\mathrm{D}}^{\mathrm{mm}}\left(P_{\mathrm{th}}\right)\right)^{-1}}{\pi\left(\frac{\eta_{\mu} P_{\mu} \beta N}{P_{\mathrm{th}}}\right)^{2 / \alpha_{\mu}}} .
$$

In particular, when only LoS mmWave links with $f_{\operatorname{Pr}}(R)=\mathbf{1}$ ( $R \leq R_{\mathrm{LoS}}$ ) are used for power transfer, the above condition is transformed as

$$
\lambda_{\mu}<\lambda_{\mathrm{mm}} \xi^{2}\left(\frac{\eta_{\mu} P_{\mu} \beta N}{P_{\mathrm{th}}}\right)^{-2 / \alpha_{\mu}},
$$

where $\xi=\min \left\{R_{\mathrm{LoS}}, \varsigma\left(\beta_{\mathrm{LoS}}^{\mathrm{mm}}, \alpha_{\mathrm{LoS}}^{\mathrm{mm}}\right)\right\}$.

Proof: Based on Corollary 1 and (14), we can directly obtain (18). By considering the LoS mmWave model with 
$f_{\operatorname{Pr}}(R)=\mathbf{1}\left(R \leq R_{\mathrm{LoS}}\right)$ [19], [26], the probability that the directed transferred power is larger than a threshold $P_{\text {th }}$ under LoS mmWave power transfer is found as

$$
\begin{aligned}
\bar{\Psi}_{\mathrm{D}}^{\mathrm{mm}} & \left(P_{\mathrm{th}}\right) \\
& =\operatorname{Pr}\left(\eta_{\mathrm{mm}} P_{\mathrm{mm}} \mathrm{M}_{\mathrm{B}} \mathrm{M}_{\mathrm{D}} L\left(\left|Y_{o}\right|\right) \mathbf{1}\left(\left|Y_{o}\right|<R_{\mathrm{LoS}}\right)>P_{\mathrm{th}}\right) \\
& \stackrel{(\mathrm{a})}{=} \int_{0}^{\xi} 2 \pi \lambda_{\mathrm{mm}} r \exp \left(-\pi \lambda_{\mathrm{mm}} r^{2}\right) d r \\
\quad= & 1-\exp \left(-\pi \lambda_{\mathrm{mm}} \xi^{2}\right)
\end{aligned}
$$

where $\xi=\min \left\{R_{\mathrm{LoS}}, \varsigma\left(\beta_{\mathrm{LoS}}^{\mathrm{mm}}, \alpha_{\mathrm{LoS}}^{\mathrm{mm}}\right)\right\}$, step (a) is obtained by considering the fact that UEs try to be connected to the nearest BSs such that there exist LoS links. Substituting (20) into (18), we obtain (19) and complete the proof.

Based on Corollary 2, we find that the mmWave tier can achieve better energy coverage than the sub- $6 \mathrm{GHz}$ tier, when the scale of sub- $6 \mathrm{GHz}$ tier is lower than the right-handside (RHS) of (18).

\section{B. Ambient RF Harvested Power}

In order to avoid singularity at zero distance and ensure the finite moments of the sum of the ambient RF signals, we incorporate the distance constraint into the path loss function in this subsection, which is $\tilde{L}(|X|)=\beta\left(\max \left(r_{o},|X|\right)\right)^{-\alpha}$ with a reference distance $r_{o}$ [33], [35]. It should be noted that the reference distance has negligible effect on the evaluation of the energy coverage probability, since the harvested energy is usually larger than the predefined threshold when $|X| \leq r_{o}$.

1) Sub-6 GHz Tier: In the sub-6 GHz tier, let $\Psi_{\mathrm{A}}^{m m}\left(P_{\mathrm{th}}\right)$ denote the probability that the ambient RF harvested power is larger than a threshold $P_{\mathrm{th}}, \bar{\Psi}_{\mathrm{A}}^{\mu}\left(P_{\mathrm{th}}\right)$ is upper bounded as

$$
\bar{\Psi}_{\mathrm{A}}^{\mu}\left(P_{\mathrm{th}}\right) \leq \min \left\{\operatorname{var}[\Xi] /\left(\frac{P_{\mathrm{th}}}{\eta_{\mu} P_{\mu}}-\mathbb{E}[\Xi]\right)^{2}, 1\right\},
$$

where $\mathbb{E}[\Xi]$ and $\operatorname{var}[\Xi]$ are respectively given by (22) and (23) at the bottom of this page. In (22) and (23), $E_{(n)}(z)=$ $\int_{1}^{\infty} e^{-z t} t^{-n} d t$ is the exponential integral [36].

Proof: Based on (1), $\bar{\Psi}_{\mathrm{A}}^{\mu}\left(P_{\mathrm{th}}\right)$ is calculated as

$$
\begin{aligned}
& \bar{\Psi}_{\mathrm{A}}^{\mu}\left(P_{\mathrm{th}}\right) \\
& \quad=\operatorname{Pr}\left(\Lambda_{\mu_{2}}>P_{\mathrm{th}}\right) \\
& =\operatorname{Pr} \underbrace{\left.\sum_{k \in \Phi_{\mu}\left(\lambda_{\mu}\right) \backslash\{o\}}\left|\mathbf{h}_{k, o} \frac{\mathbf{h}_{k}^{H}}{\left\|\mathbf{h}_{k}\right\|}\right|^{2} \tilde{L}\left(\left|X_{k, \mu}\right|\right)>\frac{P_{\mathrm{th}}}{\eta_{\mu} P_{\mu}}\right)}_{\Xi} \\
& \stackrel{(b)}{\leq} \min \left\{\frac{\operatorname{var}[\Xi]}{\left(\frac{P_{\mathrm{th}}}{\eta_{\mu} P_{\mu}}-\mathbb{E}[\Xi]\right)^{2}}, 1\right\},
\end{aligned}
$$

where step (b) is from the Chebyshev's inequality. Here, $\mathbb{E}[\Xi]$ and $\operatorname{var}[\Xi]$ denote the expectation and variance of $\Xi$, respectively.

We first derive $\mathbb{E}[\Xi]$. By using the Campbell's theorem $[37], \mathbb{E}[\Xi]$ is given by

$$
\begin{aligned}
\mathbb{E}[\Xi]=\mathbb{E} & {\left[\left|\mathbf{h}_{k, o} \frac{\mathbf{h}_{k}^{H}}{\left\|\mathbf{h}_{k}\right\|}\right|^{2}\right] \beta_{\mu} 2 \pi \lambda_{\mu} } \\
& \times \int_{0}^{\infty} \int_{r}^{\infty}\left(\max \left(r_{o}, t\right)\right)^{-\alpha_{\mu}} t f_{\left|X_{o}\right|}(r) d t d r,
\end{aligned}
$$

where $f_{\left|X_{o}\right|}(r)$ is given by (10). Since $\mathbf{h}_{k, o} \frac{\mathbf{h}_{k}^{H}}{\left\|\mathbf{h}_{k}\right\|}$ follows complex Gaussian distribution and is independent of $\mathbf{h}_{k}$, we have $\left|\mathbf{h}_{k, o} \frac{\mathbf{h}_{k}^{H}}{\left\|\mathbf{h}_{k}\right\|}\right|^{2} \sim \exp (1)$, thus $\mathbb{E}\left[\left|\mathbf{h}_{k, o} \frac{\mathbf{h}_{k}^{H}}{\left\|\mathbf{h}_{k}\right\|}\right|^{2}\right]=1$. By changing the order of integration, we further derive (25) as

$$
\begin{aligned}
\mathbb{E}[\Xi]=\beta_{\mu} 2 \pi \lambda_{\mu} \int_{0}^{\infty}\left(\max \left(r_{o}, t\right)\right)^{-\alpha_{\mu}} t \\
\times\left(1-\exp \left(-\pi \lambda_{\mu} t^{2}\right)\right) d t .
\end{aligned}
$$

After calculating the integral in (26), we obtain (22).

The variance of $\Xi$ is derived as

$$
\begin{aligned}
\operatorname{var}[\Xi]= & \left.\frac{\partial^{2}}{\partial s^{2}} \mathbb{E}[\exp (s \Xi)]\right|_{s=0}-(\mathbb{E}[\Xi])^{2} \\
\stackrel{(c)}{=} & \mathbb{E}\left[\left|\mathbf{h}_{k, o} \frac{\mathbf{h}_{k}^{H}}{\left\|\mathbf{h}_{k}\right\|}\right|^{4}\right] \beta_{\mu}^{2} 2 \pi \lambda_{\mu} \\
& \times \int_{0}^{\infty} \int_{r}^{\infty}\left(\max \left(r_{o}, t\right)\right)^{-2 \alpha_{\mu}} t f_{\left|X_{o}\right|}(r) d t d r,
\end{aligned}
$$

where step (c) is obtained by using the modified Campbell's theorem. Since $\mathbb{E}\left[\left|\mathbf{h}_{k, o} \frac{\mathbf{h}_{k}^{H}}{\left\|\mathbf{h}_{k}\right\|}\right|^{4}\right]=2$, we can finally obtain (23) and complete the proof.

2) mmWave Tier: In the mmWave tier, let $\bar{\Psi}_{\mathrm{A}}^{\mathrm{mm}}\left(P_{\mathrm{th}}\right)$ denote the probability that the ambient RF harvested power is larger than a threshold $P_{\text {th }}$, given $\varepsilon, \bar{\Psi}_{\mathrm{A}}^{\mathrm{mm}}\left(P_{\mathrm{th}}\right)>\varepsilon$ holds when the mmWave BS density meets

$$
\lambda_{\mathrm{mm}}>\left(\frac{P_{\mathrm{th}}}{\vartheta_{\mathrm{mm}} \eta_{\mathrm{mm}} P_{\mathrm{mm}}}\right)^{\frac{2}{\alpha_{\mathrm{LoS}}^{2 m}}},
$$

where $\vartheta_{\mathrm{mm}}$ is a constant value defined by

$$
\operatorname{Pr}\left(\sum_{\ell \in \Phi_{\mathrm{mm}}(1) \backslash\{0\}} G_{\ell} \Delta\left(\left|Y_{\ell, \mathrm{mm}}\right|\right)>\vartheta_{\mathrm{mm}}\right)=\varepsilon
$$

with $\Delta\left(\left|Y_{\ell, \mathrm{mm}}\right|\right)=\tilde{L}_{\mathrm{LoS}}\left(\left|Y_{\ell, \mathrm{mm}}\right|\right) \amalg\left(f_{\operatorname{Pr}}\left(\left|Y_{\ell, \mathrm{mm}}\right|\right)\right)$, where $\amalg(x)$ represents the Bernoulli distribution.

$$
\begin{aligned}
\mathbb{E}[\Xi] & =\beta_{\mu} 2 \pi \lambda_{\mu}\left(\frac{r_{o}^{-\alpha_{\mu}}}{2}\left(r_{o}^{2}-\left(\pi \lambda_{\mu}\right)^{-1}\left(1-e^{-\pi \lambda_{\mu} r_{\mathrm{o}}^{2}}\right)\right)+\frac{r_{o}^{2-\alpha_{\mu}}}{\alpha_{\mu}-2}-\frac{r_{o}^{2-\alpha_{\mu}}}{2} E_{\left(\frac{\alpha_{\mu}}{2}\right)}\left(\pi \lambda_{\mu} r_{o}^{2}\right)\right) \\
\operatorname{var}[\Xi] & =\beta_{\mu}^{2} 4 \pi \lambda_{\mu}\left(\frac{r_{o}^{-2 \alpha_{\mu}}}{2}\left(r_{\mathrm{o}}^{2}-\left(\pi \lambda_{\mu}\right)^{-1}\left(1-e^{-\pi \lambda_{\mu} r_{\mathrm{o}}^{2}}\right)\right)+\frac{r_{o}^{2-2 \alpha_{\mu}}}{2 \alpha_{\mu}-2}-\frac{r_{o}^{2-2 \alpha_{\mu}}}{2} E_{\left(\alpha_{\mu}\right)}\left(\pi \lambda_{\mu} r_{o}^{2}\right)\right)
\end{aligned}
$$


Proof: Based on (3), the probability that the ambient RF harvested power $\Lambda_{\mathrm{mm}_{2}}$ is larger than a threshold $P_{\mathrm{th}}$ can be obtained as

$$
\begin{aligned}
\bar{\Psi}_{\mathrm{A}}^{\mathrm{mm}} & \left(P_{\mathrm{th}}\right) \\
& =\operatorname{Pr}\left(\Lambda_{\mathrm{mm}_{2}}>P_{\mathrm{th}}\right) \\
& =\operatorname{Pr}\left(\sum_{\ell \in \Phi_{\mathrm{mm}}\left(\lambda_{\mathrm{mm}}\right) \backslash\{o\}} G_{\ell} \tilde{L}\left(\left|Y_{\ell, \mathrm{mm}}\right|\right)>\frac{P_{\mathrm{th}}}{\eta_{\mathrm{mm}} P_{\mathrm{mm}}}\right) .
\end{aligned}
$$

Since the ambient RF energy from the NLoS BSs is negligible, $\bar{\Psi}_{\mathrm{A}}^{\mathrm{mm}}\left(P_{\mathrm{th}}\right)$ can be lower-bounded as

$$
\begin{aligned}
& \bar{\Psi}_{\mathrm{A}}^{\mathrm{mm}}\left(P_{\mathrm{th}}\right) \\
& \geq \operatorname{Pr}\left(\sum_{\ell \in \Phi_{\mathrm{mm}}\left(\lambda_{\mathrm{mm}}\right) \backslash\{o\}} G_{\ell} \Delta\left(\left|Y_{\ell, \mathrm{mm}}\right|\right)>\frac{P_{\mathrm{th}}}{\eta_{\mathrm{mm}} P_{\mathrm{mm}}}\right) \\
& \stackrel{(d)}{=} \operatorname{Pr}\left(\sum_{\ell \in \Phi_{\mathrm{mm}}(1) \backslash\{o\}} G_{\ell} \Delta\left(\left|Y_{\ell, \mathrm{mm}}\right|\right)>\lambda_{\mathrm{mm}}^{-\frac{\alpha_{\mathrm{LoS}}^{\mathrm{mm}}}{2}} \frac{P_{\mathrm{th}}}{\eta_{\mathrm{mm}} P_{\mathrm{mm}}}\right),
\end{aligned}
$$

where step (d) is obtained by using the Mapping theorem.

Given $\varepsilon$, we define the constant $\vartheta_{\mu}$ as (29). Then we can directly obtain $\bar{\Psi}_{\mathrm{A}}^{\mu}\left(P_{\mathrm{th}}\right)>\varepsilon$ if and only if condition (28) is satisfied, which completes the proof.

Corollary 3: The ambient RF energy harvesting in the mmWave tier outperforms that in the sub- $6 \mathrm{GHz}$ tier under the condition (28), where $\vartheta_{\mathrm{mm}}$ is given by (29) with $\varepsilon=\min \left\{\operatorname{var}[\Xi] /\left(\frac{P_{\mathrm{th}}}{\eta_{\mu} P_{\mu}}-\mathbb{E}[\Xi]\right)^{2}, 1\right\}$.

It is indicated from Corollary 3 that in practice, it is still possible that the amount of ambient RF energy harvested from the mmWave tier is larger than that from the sub- $6 \mathrm{GHz}$ tier.

\section{Power Transfer Mode Selection}

In the above, we have analyzed and compared the wireless energy harvesting in the sub-6 GHz and mmWave tiers. Here, we consider mode selection for WPT in hybrid 5G scenario, i.e., UEs are at liberty to select a sub-6 $\mathrm{GHz}$ BS or mmWave BS for maximizing the directed transferred power, since the amount of harvested energy from ambient RF is much smaller compared to that from directed power transfer, see [2], [23], [24], which is also illustrated in the simulation results of section V. Thus, we have the following Propositions.

Proposition 1: The association probability that a UE selects the sub- $6 \mathrm{GHz}$ WPT is given by

$$
\begin{aligned}
\mathcal{H}_{\mu}=2 \pi & \lambda_{\mu} \int_{0}^{\infty} r \exp \left(-2 \pi \lambda_{\mathrm{mm}}\left(\int_{0}^{\hat{R}_{\mathrm{LoS}}^{\mathrm{mm}}(r)} f_{\mathrm{Pr}}(t) t d t\right.\right. \\
& \left.\left.+\int_{0}^{\hat{R}_{\mathrm{NLoS}}^{\mathrm{mm}}(r)}\left(1-f_{\mathrm{Pr}}(t)\right) t d t\right)-\pi \lambda_{\mu} r^{2}\right) d r
\end{aligned}
$$

where $\hat{R}_{\mathrm{LoS}}^{\mathrm{mm}}(r)=\left(\varpi \frac{\beta_{\mathrm{LoS}}^{\mathrm{mm}}}{\beta_{\mu}}\right)^{1 / \alpha_{\mathrm{LoS}}^{\mathrm{mm}}} r^{\frac{\alpha_{\mu}}{\alpha_{\mathrm{LoS}}^{\mathrm{mos}}}}$ and $\hat{R}_{\mathrm{NLOS}}^{\mathrm{mm}}(r)=$ $\left(\varpi \frac{\beta_{\mathrm{NLOS}}^{\mathrm{mm}}}{\beta_{\mu}}\right)^{1 / \alpha_{\mathrm{NLoS}}^{\mathrm{mm}}} r^{\frac{\alpha_{\mu}}{\alpha_{\mathrm{NL} o S}^{\mathrm{mL}}}}$ with $\varpi=\frac{\eta_{\mathrm{mm}} P_{\mathrm{mm}} \mathrm{M}_{\mathrm{B}} \mathrm{M}_{\mathrm{D}}}{\eta_{\mu} P_{\mu} N}$.

Proof: We note that in the mmWave cell, the smallscale fading is negligible, and the directed transferred power is dominated by the mmWave pathloss. In the sub- $6 \mathrm{GHz}$ cell, the small-scale fading is averaged out when the number of BS antennas is large, i.e., $\left\|\mathbf{h}_{o}\right\|^{2} \approx N$. Therefore, the probability that a UE selects the sub-6 GHz WPT can be expressed as

$$
\begin{aligned}
& \mathcal{H}_{\mu} \\
& =\operatorname{Pr}\left(\Lambda_{\mu_{1}}>\Lambda_{\mathrm{mm}_{1}}\right) \\
& =\mathbb{E}_{\left|X_{o}\right|}\left\{\operatorname{Pr}\left(\eta_{\mu} P_{\mu} N L\left(\left|X_{o}\right|\right)>\eta_{\mathrm{mm}} P_{\mathrm{mm}} \mathrm{M}_{\mathrm{B}} \mathrm{M}_{\mathrm{D}} L(R)\right)\right\} \\
& \stackrel{(e)}{=} \mathbb{E}_{\left|X_{o}\right|}[\underbrace{\operatorname{Pr}\left(R_{\ell}^{\mathrm{mm}}>\left(\varpi \frac{\beta_{\mathrm{LoS}}^{\mathrm{mm}}}{\beta_{\mu}}\right)^{1 / \alpha_{\mathrm{LoS}}^{\mathrm{mm}}}\left|X_{o}\right|^{\frac{\alpha_{\mu}}{\alpha_{\mathrm{LoS}}^{\mathrm{mm}}}} \mid \ell \in \Phi_{\mathrm{mm}}^{\mathrm{LoS}}\right)} \\
& \Theta_{\mathrm{L}}\left(\left|X_{o}\right|\right) \\
& \times \underbrace{\operatorname{Pr}\left(R_{\ell}^{\mathrm{mm}}>\left(\varpi \frac{\beta_{\mathrm{NLoS}}^{\mathrm{mm}}}{\beta_{\mu}}\right)^{1 / \alpha_{\mathrm{NLOS}}^{\mathrm{mm}}}\left|X_{o}\right|^{\frac{\alpha_{\mu}}{\alpha_{\mathrm{NLOS}}}} \mid \ell \in \Phi_{\mathrm{mm}}^{\mathrm{NLoS}}\right)}_{\Theta_{\mathrm{N}}\left(X_{o} \mid\right)}] \\
& =\int_{0}^{\infty} \Theta_{\mathrm{L}}(r) \Theta_{\mathrm{N}}(r) f_{\left|X_{o}\right|}(r) d r,
\end{aligned}
$$

where step (e) is obtained by considering two independent LoS BS process $\Phi_{\mathrm{mm}}^{\mathrm{LoS}}$ and NLoS BS process $\Phi_{\mathrm{mm}}^{\mathrm{NLoS}}, \varpi=$ $\frac{\eta_{\mathrm{mm}} P_{\mathrm{mm}} \mathrm{M}_{\mathrm{B}} \mathrm{M}_{\mathrm{D}}}{\eta_{\mu} P_{\mu} N}, f_{\left|X_{o}\right|}(r)$ is the PDF of $\left|X_{o}\right|$ given in (10). By employing the void probability, we can obtain $\Theta_{\mathrm{L}}$ as

$$
\begin{aligned}
\Theta_{\mathrm{L}}(r) & =\operatorname{Pr}\left(\text { No LoS mmWave BS closer than } \hat{R}_{\mathrm{LoS}}^{\mathrm{mm}}(r)\right) \\
& =\exp \left(-2 \pi \lambda_{\mathrm{mm}} \int_{0}^{\hat{R}_{\mathrm{LoS}}^{\mathrm{mm}}(r)} f_{\mathrm{Pr}}(t) t d t\right),
\end{aligned}
$$

where $\hat{R}_{\mathrm{LoS}}^{\mathrm{mm}}(r)=\left(\varpi \beta_{\mathrm{LoS}}^{\mathrm{mm}} / \beta_{\mu}\right)^{1 / \alpha_{\mathrm{LoS}}^{\mathrm{mm}}} r^{\alpha_{\mu} / \alpha_{\mathrm{LoS}}^{\mathrm{mm}}}$. Similar to (34), $\Theta_{\mathrm{N}}$ is given by

$$
\Theta_{\mathrm{N}}(r)=\exp \left(-2 \pi \lambda_{\mathrm{mm}} \int_{0}^{\hat{R}_{\mathrm{NLoS}}^{\mathrm{mm}}(r)}\left(1-f_{\mathrm{Pr}}(t)\right) t d t\right),
$$

where $\hat{R}_{\mathrm{NLOS}}^{\mathrm{mm}}(r)=\left(\varpi \frac{\beta_{\mathrm{NL} O \mathrm{~S}}^{\mathrm{mm}}}{\beta_{\mu}}\right)^{1 / \alpha_{\mathrm{NLOS}}^{\mathrm{mm}}} r^{\frac{\alpha_{\mu}}{\alpha_{\mathrm{NL} L S}}}$. Substituting (34) and (35) into (33), we obtain the desired result (32).

Proposition 2: The association probability that a UE selects a LoS mmWave BS for the mmWave WPT is given by

$$
\begin{aligned}
\mathcal{H}_{\mathrm{mm}}^{\mathrm{LoS}}= & 2 \pi \lambda_{\mathrm{mm}} \int_{0}^{\infty} r f_{\mathrm{Pr}}(r) \exp \left(-2 \pi \lambda_{\mathrm{mm}}\left(\int_{0}^{r} f_{\mathrm{Pr}}(t) t d t\right.\right. \\
& \left.\left.+\int_{0}^{\widetilde{R}_{\mathrm{NLOS}}^{\mathrm{mm}}(r)}\left(1-f_{\mathrm{Pr}}(t)\right) t d t\right)-\lambda_{\mu} \widetilde{A}_{\mu}(r)\right) d r,
\end{aligned}
$$

where $\widetilde{R}_{\mathrm{NLoS}}^{\mathrm{mm}}(r)=\left(\frac{\beta_{\mathrm{NLS}}^{\mathrm{mm}}}{\beta_{\mathrm{LoS}}^{\operatorname{mos}}}\right)^{1 / \alpha_{\mathrm{NLOS}}^{\mathrm{mm}}} r^{\frac{a_{\mathrm{LOS}}^{\mathrm{mm}}}{\sigma_{\mathrm{NLOS}}}}$ and $\widetilde{A}_{\mu}(r)=$ $\pi\left(\frac{\beta_{\mu}}{\varpi \beta_{\mathrm{LoS}}^{\mathrm{mim}}}\right)^{2 / \alpha_{\mu}} r^{\frac{2 \mathrm{aLOS}_{\mathrm{L}}}{\alpha_{\mu}}}$. Then the probability that a UE selects a NLoS mmWave BS for the mmWave WPT is $\mathcal{H}_{\mathrm{mm}}^{\mathrm{NLoS}}=$ $1-\mathcal{H}_{\mu}-\mathcal{H}_{\mathrm{mm}}^{\mathrm{LoS}}$.

Proof: We first define $\tau_{\mathrm{L}}$ as the probability that there exist LoS mmWave BSs. Similar to (33), the probability that a UE selects a LoS mmWave BS for the mmWave energy harvesting is calculated as

where $\Lambda_{\mathrm{mm}}^{\mathrm{LoS}}=\eta_{\mathrm{mm}} P_{\mathrm{mm}} \mathrm{M}_{\mathrm{B}} \mathrm{M}_{\mathrm{D}} \beta_{\mathrm{LoS}}^{\mathrm{mm}}\left|Y_{o}\right|^{-\alpha_{\mathrm{LoS}}^{\mathrm{mm}}}$ is the directed transferred power from the nearest LoS mmWave BS, and the PDF of $\left|Y_{o}\right|$ is given by [19]

$$
f_{\left|Y_{o}\right|}(r)=\frac{2 \pi \lambda_{\mathrm{mm}}}{\tau_{\mathrm{L}}} r f_{\operatorname{Pr}}(r) e^{-2 \pi \lambda_{\mathrm{mm}} \int_{0}^{r} f_{\mathrm{Pr}}(t) t d t} .
$$




$$
\begin{aligned}
& \mathcal{H}_{\mathrm{mm}}^{\mathrm{LoS}}=\tau_{\mathrm{L}} \mathbb{E}_{\left|Y_{o}\right|}\{\underbrace{\operatorname{Pr}\left(\Lambda_{\mathrm{mm}_{1}}^{\mathrm{LoS}}>\Lambda_{\mu_{1}}\right)}_{\Theta_{\mu}\left(\left|Y_{o}\right|\right)} \\
& \times \underbrace{\operatorname{Pr}\left(\Lambda_{\mathrm{mm}_{1}}^{\mathrm{LoS}}>\eta_{\mathrm{mm}} P_{\mathrm{mm}} \mathrm{M}_{\mathrm{B}} \mathrm{M}_{\mathrm{D}} \beta_{\mathrm{NLoS}}^{\mathrm{mm}}\left(R_{\ell}^{\mathrm{mm}}\right)^{-\alpha_{\mathrm{NLoS}}^{\mathrm{mm}}} \mid \ell \in \Phi_{\mathrm{mm}}^{\mathrm{NLoS}}\right)}_{\Theta_{\mathrm{N}}\left(\left|Y_{o}\right|\right)}\} \\
& =\tau_{\mathrm{L}} \int_{0}^{\infty} \Theta_{\mu}(r) \bar{\Theta}_{\mathrm{N}}(r) f_{\left|Y_{o}\right|}(r) d r,
\end{aligned}
$$

Similar to (34), $\Theta_{\mu}(r)$ is derived as

$$
\begin{aligned}
\Theta_{\mu}(r) & =\operatorname{Pr}\left(\eta_{\mathrm{mm}} P_{\mathrm{mm}} \mathrm{M}_{\mathrm{B}} \mathrm{M}_{\mathrm{D}} \beta_{\mathrm{LoS}}^{\mathrm{mm}} r^{-\alpha_{\mathrm{LoS}}^{\mathrm{mm}}}>\eta_{\mu} P_{\mu} N \beta_{\mu} R_{\mu}^{-\alpha_{\mu}}\right) \\
& =\operatorname{Pr}\left(R_{\mu}>\left(\frac{\beta_{\mu}}{\varpi \beta_{\mathrm{LoS}}^{\mathrm{mm}}}\right)^{1 / \alpha_{\mu}} r^{\frac{a_{\mathrm{LoS}}^{\mathrm{mm}}}{\alpha_{\mu}}}\right) \\
& =\exp \left(-\lambda_{\mu} \widetilde{A}_{\mu}(r)\right),
\end{aligned}
$$

where $\widetilde{A}_{\mu}(r)=\pi\left(\frac{\beta_{\mu}}{\varpi \beta_{\mathrm{LoS}}^{\mathrm{mm}}}\right)^{2 / \alpha_{\mu}} r^{\frac{2 \alpha_{\mathrm{LoS}}^{\mathrm{mm}}}{\alpha_{\mu}}}$. Then $\bar{\Theta}_{\mathrm{N}}(r)$ is similarly derived as

$$
\bar{\Theta}_{\mathrm{N}}(r)=\exp \left(-2 \pi \lambda_{\mathrm{mm}} \int_{0}^{\widetilde{R}_{\mathrm{NLOS}}^{\mathrm{mm}}(r)}\left(1-f_{\mathrm{Pr}}(t)\right) t d t\right)
$$

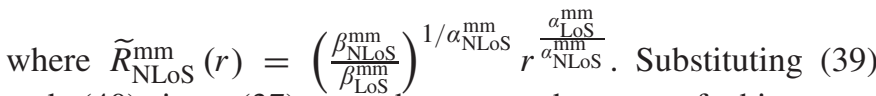
and (40) into (37), as shown at the top of this page, we obtain (36) and complete the proof.

When only LoS mmWave links with $f_{\mathrm{Pr}}(R)=$ $\mathbf{1}\left(R \leq R_{\mathrm{LoS}}\right)$ are able to transfer energy by mmWave radiation, the association probability that a UE selects the sub-6 GHz WPT given by (32) reduces to

$$
\tilde{\mathcal{H}}_{\mu}=2 \pi \lambda_{\mu} \int_{0}^{\infty} r \exp \left(-\pi \lambda_{\mathrm{mm}}(\hat{\hat{R}}(r))^{2}-\pi \lambda_{\mu} r^{2}\right) d r
$$

where $\hat{R}(r)=\min \left(\left(\mathrm{M}_{\mathrm{B}} \mathrm{M}_{\mathrm{D}}\right)^{1 / \alpha_{\mathrm{LoS}}^{\mathrm{mm}}}\left(\frac{\eta_{\mathrm{mm}} P_{\mathrm{mm}} \beta_{\mathrm{LOS}}^{\mathrm{mm}}}{\eta_{\mu} P_{\mu} N \beta_{\mu}}\right)^{1 / \alpha_{\mathrm{LoS}}^{\mathrm{mm}}}\right.$

$\left.r^{\frac{\alpha_{\mu}}{\alpha_{\operatorname{LoS}}^{\alpha m}}}, R_{\mathrm{LoS}}\right)$. Accordingly, the association probability that a UE selects a LoS mmWave BS for the mmWave WPT is $\widetilde{\mathcal{H}}_{\mathrm{mm}}^{\mathrm{LoS}}=1-\widetilde{\mathcal{H}}_{\mu}$. In light of $\alpha_{\mathrm{LoS}}^{\mathrm{mm}} \geq 2$ [11], we find that compared to increasing the mmWave antenna gain $\mathrm{M}_{\mathrm{B}} \mathrm{M}_{\mathrm{D}}$, $\widetilde{\mathcal{H}}_{\mathrm{mm}}^{\mathrm{LoS}}$ grows at a higher speed when increasing the mmWave density $\lambda_{\mathrm{mm}}$, which highlights the importance of achieving more mmWave BS densification gains for mmWave WPT.

\section{Throughrut Analysis}

The previous section has revealed how many small cells are required for obtaining the expected harvested energy. In this section, we characterize the uplink performance in terms of the throughput in the considered networks. We will calculate how many small cells should be deployed in the sub- $6 \mathrm{GHz}$ and mmWave tiers, in order to achieve the targeted throughput. Moreover, we will answer how many mmWave cells are needed to outperform a specific sub- $6 \mathrm{GHz}$ tier. We assume that in each block time $T$, UEs first harvest RF energy with the time duration $\tau T(0<\tau<1)$, and the rest of time is allocated for uplink transmissions by fully using the harvested energy.

\section{A. Sub-6 GHz Tier}

In the sub- $6 \mathrm{GHz}$ tier, we define the ratio between the sub-6 GHz BS density and active $\mu \mathrm{UE}$ density as $\kappa_{\mu \mathrm{UE}}=\lambda_{\mu} / \widetilde{\lambda}_{\mu \mathrm{UE}}$, to characterize the tier scale. For a given active $\mu \mathrm{UE}$ density, larger $\kappa_{\mu \mathrm{UE}}$ means that the network is denser and the distance between a $\mu \mathrm{UE}$ and its serving BS is shorter, which brings more BS densification gains. Note that the achievable BS densification gain can help combat the uplink interference without using complicated interference coordination methods such as [30]. For a given sub-6 GHz BS density, lower $\kappa_{\mu \mathrm{UE}}$ means that more active $\mu \mathrm{UEs}$ are served, which results in larger uplink interference.

We first derive the exact expression for the throughput $\mathrm{C}_{\mu}$ between a typical $\mu \mathrm{UE}$ and its serving sub-6 $\mathrm{GHz}$ BS as

$$
\mathrm{C}_{\mu}=\frac{1-\tau}{\ln 2} \mathrm{BW}_{\mu} \int_{0}^{\infty} \frac{\varphi_{\mu}(t)}{t} e^{-\tau_{\mu \mu \mathrm{UE}}^{\frac{-\alpha_{\mu}}{2}} \sigma^{2} t} d t,
$$

where $\mathrm{BW}_{\mu}$ is the sub-6 $\mathrm{GHz}$ bandwidth, $\varphi_{\mu}(t)$ is given by (43) (see top of the next page), in which $\hbar_{\mu}=\frac{\tau}{1-\tau} \eta_{\mu} P_{\mu} N \lambda_{\mu}^{\frac{\alpha_{\mu}}{2}}$. with

Proof: See Appendix A.

To further shed light on the effect of $\kappa_{\mu} \mathrm{UE}$ on the throughput, we provide a closed-form lower bound expression for (42), which is given by

$$
\begin{aligned}
\mathrm{C}_{\mu}^{\mathrm{L}}= & (1-\tau) \mathrm{BW}_{\mu} \\
& \times \log _{2}\left(1+\kappa_{\mu \mathrm{UE}}^{\frac{\alpha_{\mu}}{2}} \frac{N e^{\alpha_{\mu} e^{\pi r_{o}^{2}} \zeta_{o}}}{2 \pi^{2} \zeta_{1} e^{\pi r_{o}^{2}} r_{o}^{2-\alpha_{\mu}} E_{\left(\frac{\alpha_{\mu}}{2}\right)}\left(r_{o}^{2} \pi\right)}\right),
\end{aligned}
$$

where $\zeta_{o}=E i\left(-r_{o}^{2} \pi\right)-2 e^{-r_{o}^{2} \pi} \ln r_{o}, \zeta_{1}=\frac{r_{o}^{2-\alpha_{\mu}}}{\alpha_{\mu}-2}$, and $E i(\cdot)$ is the exponential integral function [36, eq. (8.211)].

Proof: See Appendix B.

It is explicitly shown from (45) that the throughput increases with $\kappa_{\mu} \mathrm{UE}$ and $N$, which means that adding more small cells or antennas enhances the performance. Also, for large $\kappa_{\mu} \mathrm{UE}$ or $N$, the throughput scales as $(1-\tau)$ $\mathrm{BW}_{\mu}\left(\frac{\alpha_{\mu}}{2} \log _{2}\left(\kappa_{\mu \mathrm{UE}}\right)+\log _{2}(N)\right)$, which indicates that the 


$$
\begin{gathered}
\varphi_{\mu}(t)=\frac{2 \pi}{e^{-\pi r_{o}}} \widetilde{I}_{\mu}(t) \int_{r_{o}}^{\infty}\left(1-e^{-t \kappa_{\mu \mathrm{UE}}^{\frac{\alpha_{\mu}}{2}} N \hbar_{\mu} L^{2}(r)}\right) r e^{-\pi r^{2}} d r \\
\widetilde{I}_{\mu}(t)=\exp \left\{-\frac{4 \pi^{2}}{e^{-\pi r_{o}^{2}}} \int_{r_{o}}^{\infty} \int_{r_{o}}^{\infty} \frac{t \hbar_{\mu} L(y) L(x)}{1+t \hbar_{\mu} L(y) L(x)} x y e^{-\pi y^{2}} d y d x\right\}
\end{gathered}
$$

throughput grows at a higher speed when increasing $\kappa_{\mu \mathrm{UE}}$, compared to increasing the number of BS antennas. ${ }^{3}$

Corollary 4: An expected throughput $C_{\text {th }}^{\mu}$ is achievable when the ratio between the sub- $6 \mathrm{GHz}$ BS density and active $\mu$ UE density satisfies

$$
\begin{aligned}
& \kappa_{\mu \mathrm{UE}} \\
& \geq N^{-\frac{2}{\alpha_{\mu}}}\left(\frac{\left(2^{\frac{\mathrm{C}_{h}^{\mu}}{\mathrm{BW}(1-\tau)}}-1\right) 2 \pi^{2} \zeta_{1} e^{\pi r_{o}^{2}} r_{o}^{2-\alpha_{\mu}} E_{\left(\frac{\alpha_{\mu}}{2}\right)}\left(r_{o}^{2} \pi\right)}{e^{\alpha_{\mu} e^{\pi r_{o}^{2}} \zeta_{o}}}\right)^{\frac{2}{\alpha_{\mu}}} .
\end{aligned}
$$

From Corollary 4, the ratio between the sub-6 GHz BS density and the active $\mu \mathrm{UE}$ density should be larger than a critical value for obtaining the desired performance.

\section{B. mmWave Tier}

In the mmWave tier, we similarly define the ratio between the mmWave BS density and the active mmUE density as $\kappa_{\mathrm{mmUE}}=\lambda_{\mathrm{mm}} / \widetilde{\lambda}_{\mathrm{mmUE}}$. The throughput can be derived as

$$
C_{\mathrm{mm}}=\frac{(1-\tau)}{\ln 2} \mathrm{BW}_{\mathrm{mm}} \int_{0}^{\infty} \frac{\varphi_{\mathrm{mm}}(t)}{t} e^{-\sigma^{2} t} d t
$$

where $\mathrm{BW}_{\mathrm{mm}}$ denotes the mmWave bandwidth, $\hbar_{\mathrm{mm}}=$ $\frac{\tau}{1-\tau} \eta_{\mathrm{mm}} P_{\mathrm{mm}} \mathrm{M}_{\mathrm{B}} \mathrm{M}_{\mathrm{D}}$, and $\varphi_{\mathrm{mm}}(t)$ is given by (48), as shown at the bottom of this page. where (49), as shown at the bottom of this page, with $\phi(y)$, as shown at the bottom of this page.

Proof: See Appendix C.

In the wireless powered mmWave tier, the transmit power of a mmUE is much lower due to the limited harvested energy resulting from the energy loss of propagation and the RF-to-DC conversion, which means that it is more likely to be noise-limited in the wireless powered uplink mmWave tier. As such, it is necessary to analyze the throughput in the noise-limited mmWave scenario, where uplink interference is negligible. Note that such analysis can also be viewed as a tight upper bound of the exact throughput given by (47), and has a

\footnotetext{
${ }^{3}$ Note that the pathloss exponent $\alpha_{\mu} \geq 2$ in the practical environments [11].
}

good approximation to (47) in the practical urban settings [20]. Therefore, the throughput expression in (47) can be further simplified as

$$
\begin{aligned}
C_{\mathrm{mm}}^{\mathrm{n}}=(1- & \tau) \mathrm{BW}_{\mathrm{mm}} \times \int_{r_{o}}^{R_{\mathrm{LoS}}} \log _{2} \\
& \times\left(1+\hbar_{\mathrm{mm}}\left(\mathrm{M}_{D} \mathrm{M}_{B} \beta_{\mathrm{LoS}}^{\mathrm{mm}}\right)^{2} \frac{r^{-2 \alpha_{\mathrm{LoS}}^{\mathrm{mm}}}}{\sigma^{2}}\right) \widetilde{f}_{|Y|}(r) d r,
\end{aligned}
$$

where $\widetilde{f}_{|Y|}(\cdot)=\frac{2 \pi \lambda_{\mathrm{mm}} r}{e^{-\pi \lambda_{\mathrm{mm}} r_{o}^{2}}} \exp \left(-\pi \lambda_{\mathrm{mm}} r^{2}\right)$ is the modified PDF of the distance $|Y|$ between a mmUE and its nearest mmWave BS under the constraint $|Y| \geq r_{o}$.

We next derive a closed-form lower bound expression for $(50)$ as

$$
C_{\mathrm{mm}}^{\mathrm{L}}=(1-\tau) \mathrm{BW}_{\mathrm{mm}} \log _{2}\left(1+\frac{e^{\widetilde{\varphi}_{\mathrm{mm}}\left(\lambda_{\mathrm{mm}}\right)}}{\sigma^{2}}\right) .
$$

In (51), $\widetilde{\varphi}_{\mathrm{mm}}\left(\lambda_{\mathrm{mm}}\right)$ is an increasing function of $\lambda_{\mathrm{mm}}$, which is given by

$$
\begin{aligned}
\widetilde{\varphi}_{\mathrm{mm}} & \left(\lambda_{\mathrm{mm}}\right) \\
= & \left(\ln \left(\hbar_{\mathrm{mm}} \mathrm{M}_{D} \mathrm{M}_{B}\right)+2\left(\ln \beta_{\mathrm{LoS}}^{\mathrm{mm}}+\frac{\alpha_{\mathrm{LoS}}^{\mathrm{mm}}}{2} \ln \left(\pi \lambda_{\mathrm{mm}}\right)\right)\right) \\
& \times\left(e^{-a_{1}}-e^{-b_{1}}\right)-\alpha_{\mathrm{LoS}}^{\mathrm{mm}}\left(E i\left(-b_{1}\right)\right. \\
& \left.+\Gamma\left(0, a_{1}\right)+e^{-a_{1}} \ln \left(a_{1}\right)-e^{-b_{1}} \ln b_{1}\right)
\end{aligned}
$$

with $a_{1}=\pi \lambda_{\mathrm{mm}} r_{o}^{2}$ and $b_{1}=\pi \lambda_{\mathrm{mm}} R_{\mathrm{LoS}}^{2}$.

Proof: See Appendix D.

Corollary 5: Based on (51), we find that the expected throughput $C_{\mathrm{th}}^{\mathrm{mm}}$ can be achieved when mmWave density $\lambda_{\mathrm{mm}}$ satisfies the following condition:

$$
\lambda_{\mathrm{mm}} \geq \widetilde{\varphi}_{\mathrm{mm}}^{-1}\left(\ln \sigma^{2}\left(2^{\frac{c_{\mathrm{th}}^{\mathrm{mm}}}{(1-\tau) \mathrm{BW} m \mathrm{~mm}}}-1\right)\right),
$$

where $\widetilde{\varphi}_{\mathrm{mm}}^{-1}$ is the inverse function of $\widetilde{\varphi}_{\mathrm{mm}}$.

$$
\begin{aligned}
& \varphi_{\mathrm{mm}}(t)=\frac{2 \pi \lambda_{\mathrm{mm}}}{e^{-\pi \lambda_{\mathrm{mm}} r_{o}}} \widetilde{I}_{\mathrm{mm}}(t) \int_{r_{o}}^{R_{\mathrm{LoS}}}\left(1-e^{-t \hbar_{\mathrm{mm}} \mathrm{M}_{D} \mathrm{M}_{B}\left(\beta_{\mathrm{LoS}}^{\mathrm{mm}}\right)^{2} r^{-2 a_{\mathrm{LoS}}^{\mathrm{mm}}}}\right) r \exp \left(-\pi \lambda_{\mathrm{mm}} r^{2}\right) d r, \\
& \tilde{I}_{\mathrm{mm}}(t)=\exp \left\{-2 \pi \lambda_{\mathrm{mm}} \kappa_{\mathrm{mmUE}}^{-1} \int_{r_{o}}^{R_{\mathrm{LoS}}}(1-\phi(y)) y d y\right\} \\
& \phi(y)=\frac{2 \pi \lambda_{\mathrm{mm}}}{e^{-\pi \lambda_{\mathrm{mm}} r_{o}^{2}}} \sum_{\ell \in\left\{\mathrm{M}_{\mathrm{B}}, \mathrm{m}_{\mathrm{B}}\right\}} \sum_{k \in\left\{\mathrm{M}_{\mathrm{D}}, \mathrm{m}_{\mathrm{D}}\right\}} \operatorname{Pr}_{\ell k} \int_{r_{o}}^{R_{\mathrm{LoS}}} e^{-t \hbar_{\mathrm{mm}} \ell k}\left(\beta_{\mathrm{LoS}}^{\mathrm{mm}}\right)^{2}(z y)^{-2 a_{\mathrm{LoS}}^{\mathrm{mm}}} z \exp \left(-\pi \lambda_{\mathrm{mm}} z^{2}\right) d z
\end{aligned}
$$


TABLE I

SimUlation PARAMETERS

\begin{tabular}{|l|c|}
\hline Parameter & Value \\
\hline MmWave carrier frequency & $f_{\mathrm{mm}}=28 \mathrm{GHz}$ \\
\hline RF-to-DC conversion efficiency & $\eta_{\mu}=\eta_{\mathrm{mm}}=0.6$ \\
\hline BS transmit power & $P_{\mu}=P_{\mathrm{mm}}=30 \mathrm{dBm}$ \\
\hline Reference distance & $r_{o}=1$ \\
\hline MmWave pathloss exponent & $\alpha_{\mathrm{LoS}}^{\mathrm{mm}}=2, \alpha_{\mathrm{NLoS}}^{\mathrm{mm}}=2.9$ \\
\hline
\end{tabular}

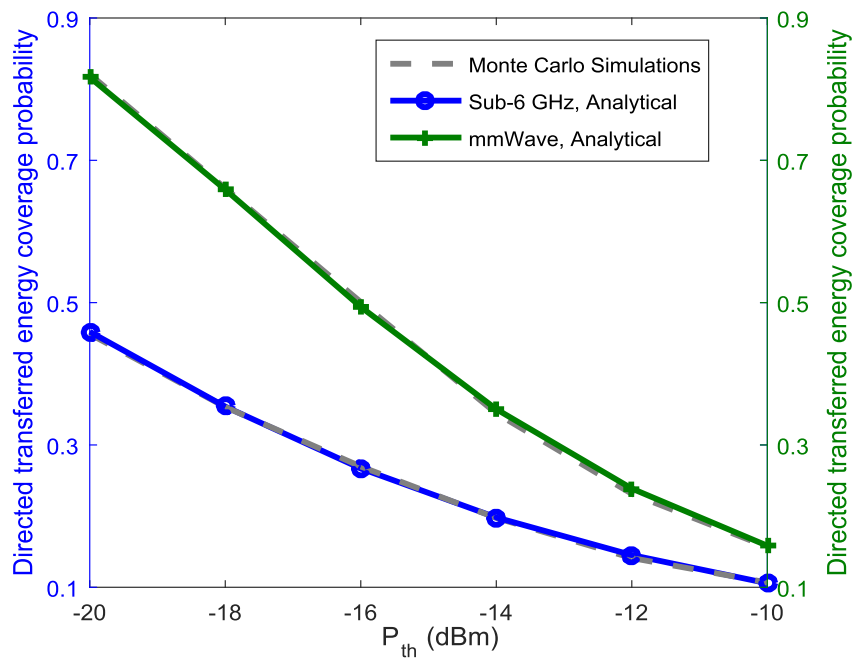

Fig. 1. Directed transferred energy coverage probability with $N=32$, $\alpha_{\mu}=2.7, \lambda_{\mu}=0.002$ and $\lambda_{\mathrm{mm}}=0.02$.

It is confirmed by Corollary $\mathbf{5}$ that the mmWave density needs to be larger than the RHS of (53), in order to achieve a targeted throughput.

Corollary 6: Based on Corollary 4, a $\mu \mathrm{UE}$ can achieve a higher throughput than a mmUE when $\kappa_{\mu \mathrm{UE}}$ satisfies (46) with $C_{\mathrm{th}}^{\mu}=C_{\mathrm{mm}}$ given in (47). Based on Corollary 5, a mmUE can achieve a higher throughput than a $\mu \mathrm{UE}$ when $\lambda_{\mathrm{mm}}$ satisfies (53) with $C_{\mathrm{th}}^{\mathrm{mm}}=\mathrm{C}_{\mu}$ given in (42).

Corollary 6 confirms that whether the sub-6 GHz tier performs better than the mmWave tier depends on the number of sub- $6 \mathrm{GHz}$ small cells in the $5 \mathrm{G}$ networks.

\section{Simulation Results}

In this section, we present simulation results to show the energy coverage and throughput performance in wireless powered 5G dense cellular networks. The results validate the prior analysis, and further illustrate the impacts of node density on the RF energy harvesting and information transmission. The basic simulation parameters are shown in Table I.

\section{A. Energy Coverage}

In this subsection, we study energy coverage in the sub- $6 \mathrm{GHz}$ and mmWave tiers. It is assumed that the LoS prob-

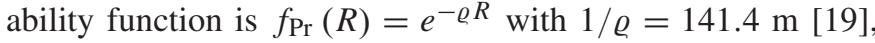
the sub- $6 \mathrm{GHz}$ carrier frequency is $f_{c}=1.5 \mathrm{GHz}$, and the mmWave antenna beam pattern at a mmWave BS and mmUE are $\left(\mathrm{M}_{\mathrm{B}}, \mathrm{m}_{\mathrm{B}}, \theta_{\mathrm{B}}\right)=\left(18 \mathrm{~dB},-2 \mathrm{~dB}, 10^{\circ}\right)$ and $\left(\mathrm{M}_{\mathrm{D}}, \mathrm{m}_{\mathrm{D}}, \theta_{\mathrm{D}}\right)=$ $\left(10 \mathrm{~dB},-10 \mathrm{~dB}, 45^{\circ}\right)$, respectively.

Fig. 1 shows the directed transferred energy coverage probability results in the sub-6 GHz and mmWave tiers. The

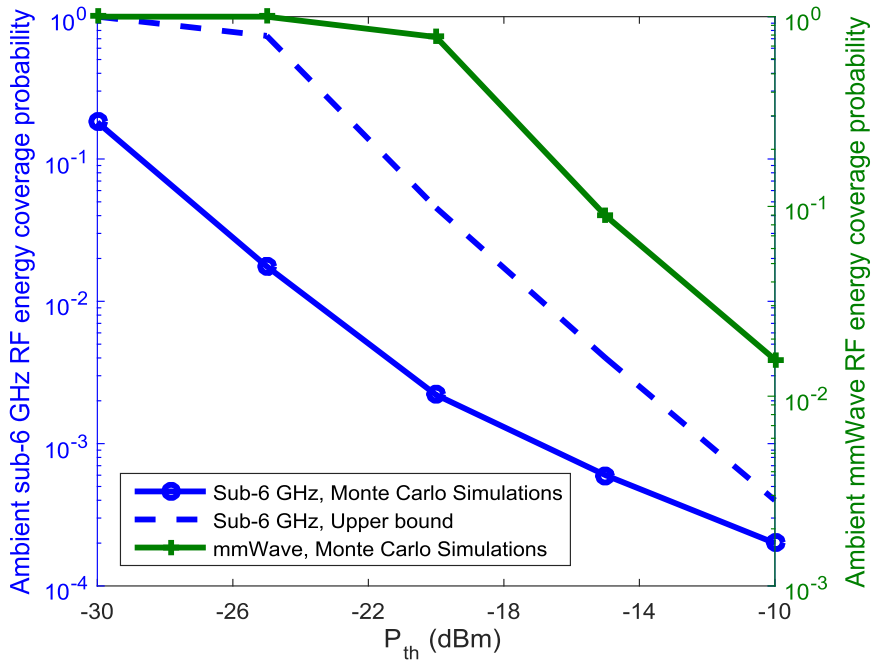

Fig. 2. Ambient RF energy coverage probability with $\alpha_{\mu}=2.6, \lambda_{\mu}=0.002$ and $\lambda_{\mathrm{mm}}=0.5$.

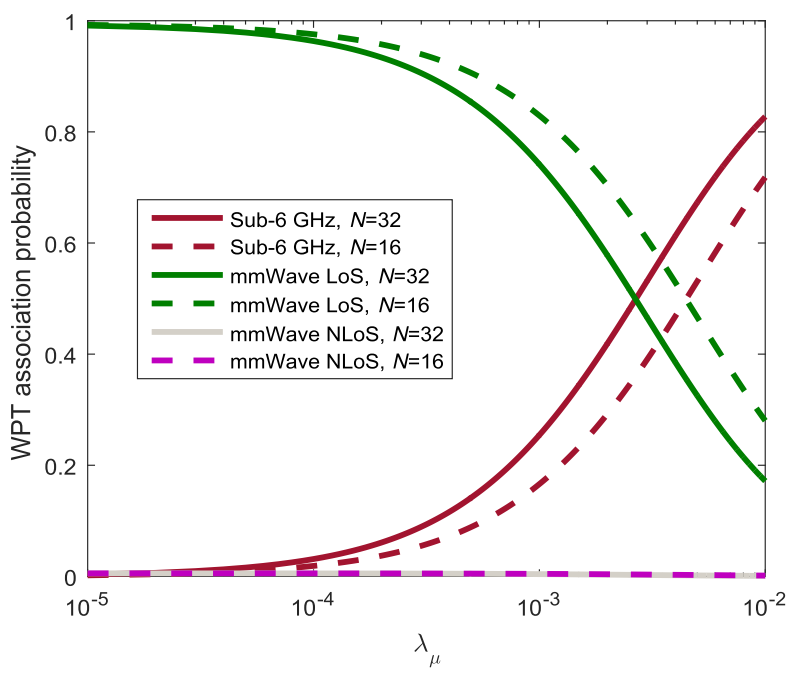

Fig. 3. WPT association probability with $\alpha_{\mu}=2.7$ and $\lambda_{\mathrm{mm}}=0.01$.

analytical results are obtained from (13) and (14), respectively, which are validated by Monte Carlo simulations. The result in (13) can predict the energy coverage of the sub-6 GHz tier. MmWave power transfer can be better than the sub- $6 \mathrm{GHz}$ counterpart, due to the mmWave directivity gain and densification gain.

Fig. 2 shows the ambient RF energy coverage probability results in the sub- $6 \mathrm{GHz}$ and mmWave tiers. We observe that for ultra-dense mmWave tier, the harvested ambient mmWave RF energy can still be larger than that in the sub- $6 \mathrm{GHz}$ tier with comparably lower BS density. Compared to Fig. 1, it is indicated that the amount of harvested energy from the ambient $\mathrm{RF}$ is much lower than the directed power transfer.

Fig. 3 demonstrates the association probability that a UE selects a sub-6 GHz BS or mmWave BS in hybrid cellular networks. The results are obtained based on Proposition 1 and Proposition 2. We observe that the association probability for sub-6 GHz WPT increases with the sub- $6 \mathrm{GHz}$ BS density because of obtaining higher densification gains, and it will 


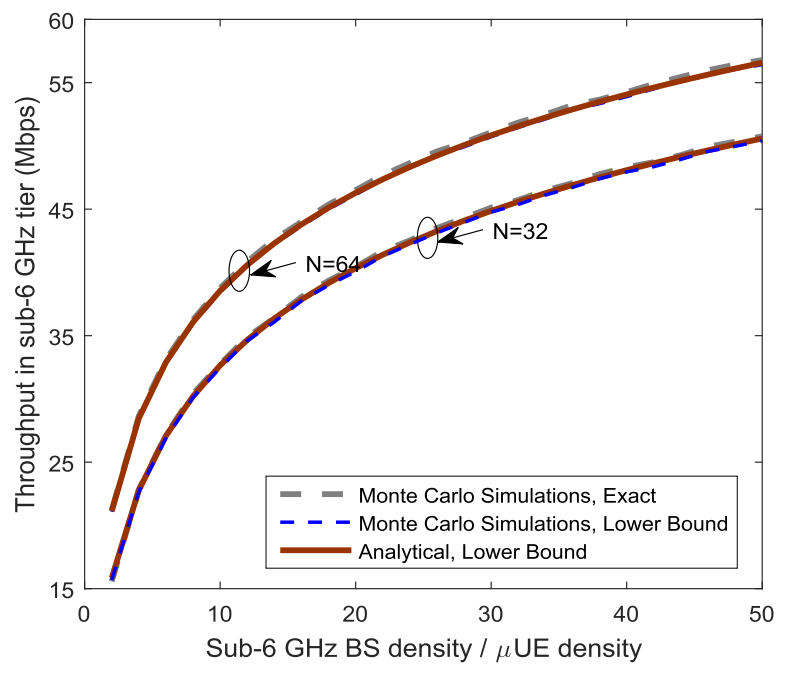

Fig. 4. Throughput in sub-6 GHz tier with $\tilde{\lambda}_{\mu \mathrm{UE}}=0.001$.

also be improved by adding sub- $6 \mathrm{GHz}$ antennas for achieving more antenna gains. More UEs will select mmWave BSs to transfer energy when the sub-6 GHz BS density is much lower than the mmWave BS density, which implies that dense small cells are needed to shorten the energy transfer distance between the UE and its associated BS. Compared to the mmWave LoS, the association probability that a UE selects a mmWave NLoS power transfer is negligible.

\section{B. Throughput}

Here, we study the impact of the ratio between the BS density and the active UE density on the throughput. In the simulations, the energy harvesting time allocation factor is $\tau=0.7$, the sub- $6 \mathrm{GHz}$ carrier frequency is $f_{c}=1 \mathrm{GHz}$, the sub- $6 \mathrm{GHz}$ pathloss exponent is $\alpha_{\mu}=2.6$, the sub- $6 \mathrm{GHz}$ bandwidth is $\mathrm{BW}_{\mu}=20 \mathrm{MHz}$, the mmWave bandwidth is $\mathrm{BW}_{\mathrm{mm}}=1 \mathrm{GHz}$, and the mmWave antenna beam pattern at an active mmUE and a mmWave $\mathrm{BS}$ are $\left(\mathrm{M}_{\mathrm{D}}, \mathrm{m}_{\mathrm{D}}, \theta_{\mathrm{D}}\right)=\left(3 \mathrm{~dB},-3 \mathrm{~dB}, 45^{\circ}\right)$ and $\left(\mathrm{M}_{\mathrm{B}}, \mathrm{m}_{\mathrm{B}}, \theta_{\mathrm{B}}\right)=\left(18 \mathrm{~dB},-2 \mathrm{~dB}, 10^{\circ}\right)$, respectively, and the maximum LoS distance is $R_{\mathrm{LoS}}=20 \mathrm{~m}$. The noise power is obtained by $\sigma^{2}=-174+10 \log 10(\mathrm{BW})+\mathrm{Nf} \mathrm{dBm}$ with $7 \mathrm{~dB}$ noise figure $(\mathrm{Nf})$.

Fig. 4 shows the throughput in the sub- $6 \mathrm{GHz}$ tier. The analytical lower bound curves are obtained from (45), which tightly matches with the simulated exact curves. We see that deploying more sub-6 GHz small cells can significantly increase the throughput, due to the densification gain. Adding more BS antennas can further enhance the spectrum efficiency and bring an increase in throughput. It is also indicated from Fig. 4 that given a specific throughput level, the required number of small cells can be cut by using more antennas at each BS.

Fig. 5 illustrates the throughput in the mmWave tier. The simulated throughput curves based on the SINR has a good match with that based on SNR, confirming that the wireless powered uplink mmWave tier is noise-limited. The analytical lower bounds are obtained from (51), which can well approximate the simulated exact curves. We find that adding more sub-6 GHz small cells has a substantial increase in throughput.

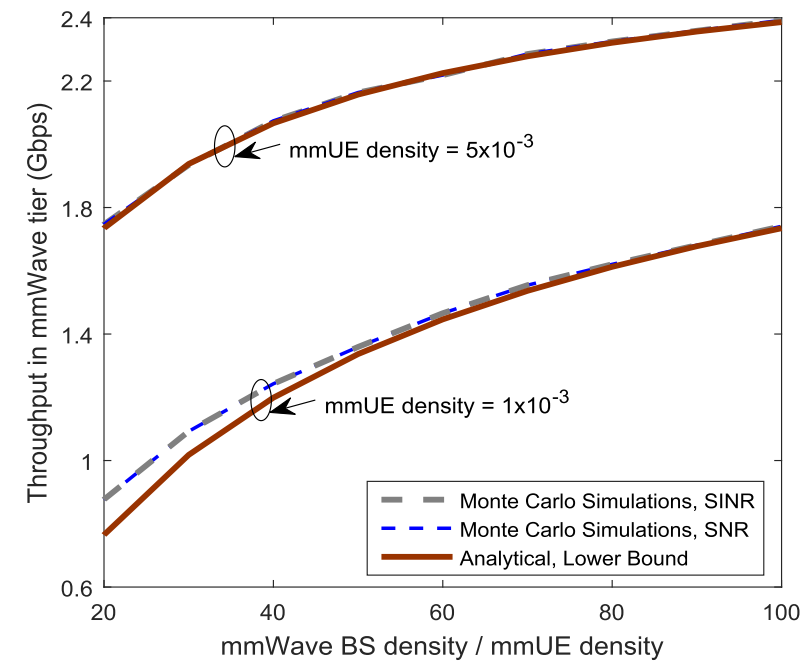

Fig. 5. Throughput in mmWave tier.

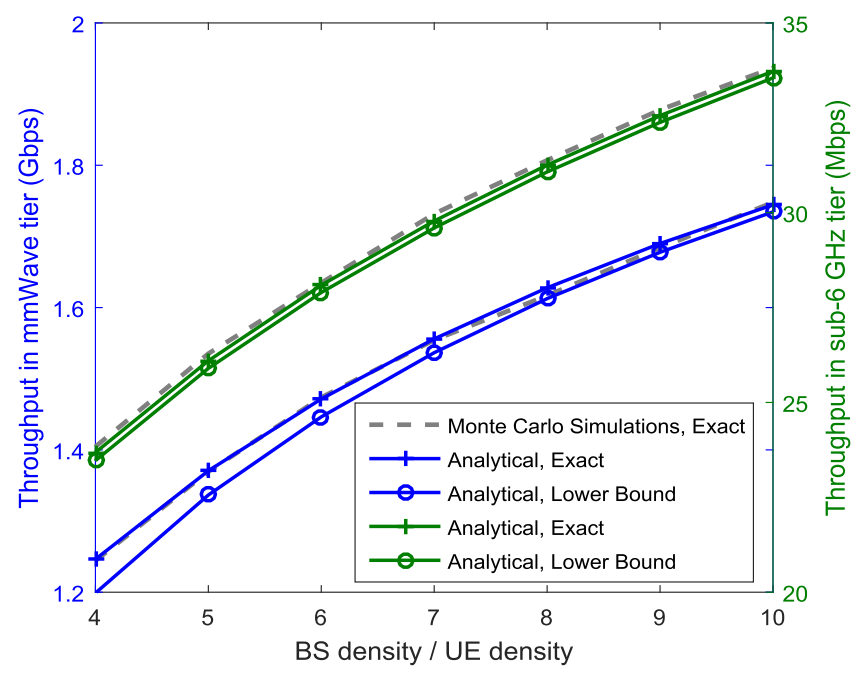

Fig. 6. Throughput comparison between mmWave tier and sub-6 GHz tier for the same tier scale with $\widetilde{\lambda}_{\mu \mathrm{UE}}=\widetilde{\lambda}_{\mathrm{mmUE}}=0.01$.

Fig. 6 compares the throughput between the mmWave tier and the sub- $6 \mathrm{GHz}$ tier for the same scale, i.e., same numbers of BSs and active UEs. The analytical exact and lower bounds of the mmWave tier are obtained from (47) and (51), respectively. The analytical exact and lower bounds of the sub$6 \mathrm{GHz}$ tier are obtained from (42) and (45), respectively. Our analysis is validated by the simulated results. It is implied that the Gbps transmission rate is still likely to be achieved in the wireless powered dense mmWave tier, which significantly outperforms the sub- $6 \mathrm{GHz}$ tier. Moreover, in the wireless powered ultra-dense mmWave scenarios, interference is still negligible, i.e., noise-limited.

Fig. 7 provides the throughput comparison for different mmWave carrier frequencies. The LoS pathloss exponent is set as 2 at $28 \mathrm{GHz}, 38 \mathrm{GHz}$ and $73 \mathrm{GHz}$, and its value is 2.25 at $60 \mathrm{GHz}$ [38], [39]. We observe that the achievable throughput is the highest at $28 \mathrm{GHz}$ since it has the lowest propagation loss. The performance gap between different mmWave carrier frequencies is larger when increasing the BS density, which indicates that lower mmWave frequencies can obtain more 


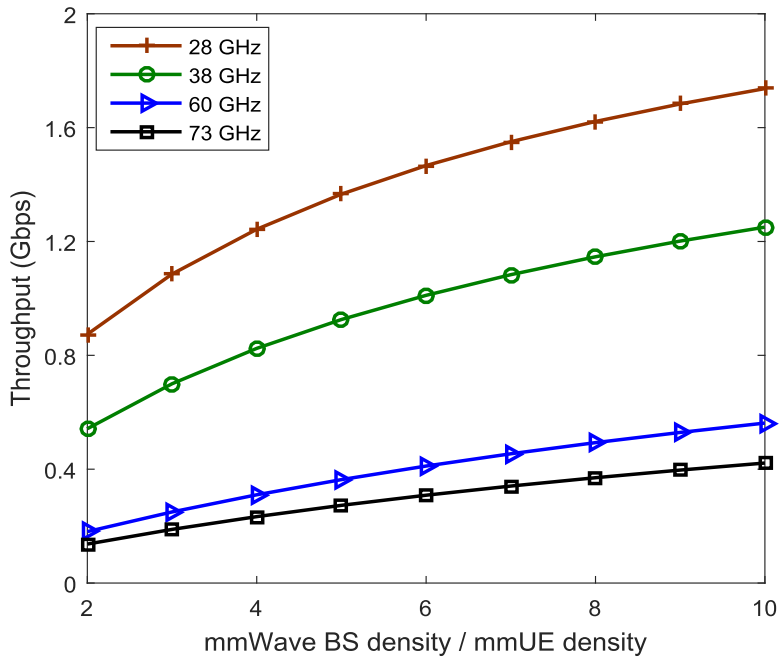

Fig. 7. Throughput comparison for different mmWave carrier frequencies with $\widetilde{\lambda}_{\text {mmUE }}=0.01$.

densification gains. In addition, the performance at $73 \mathrm{GHz}$ is close to that at $60 \mathrm{GHz}$, due to the fact that the atmospheric absorption at $60 \mathrm{GHz}$ is more severe than that at $73 \mathrm{GHz}$, which leads to higher LoS pathloss exponent at $60 \mathrm{GHz}$.

\section{CONCLUSION}

This paper studied WPT in dense cellular networks, where a large number of sub- $6 \mathrm{GHz}$ and mmWave BSs with antenna arrays are deployed to power UEs. The expressions of the energy coverage probability were derived, in order to provide comparisons between the sub-6 GHz and mmWave energy harvesting. We obtained the BS density condition when the mmWave tier can provide more RF energy than the sub- $6 \mathrm{GHz}$ tier. In addition, the probability that a UE selects the sub$6 \mathrm{GHz}$ or mmWave power transfer was quantified. We then derived the throughput in the uplink sub- $6 \mathrm{GHz}$ and mmWave tiers. We obtained the number of small cells that are required to achieve a targeted level of throughput. Also, we presented the BS density conditions when the mmWave UE achieves higher throughput than the sub-6 GHz UE.

\section{APPENDIX A}

\section{A Detailed Derivation of (42)}

According to the Mapping theorem [37], (4) can be written as

$$
\operatorname{SINR}_{\mu}=\frac{\lambda_{\mu}^{\frac{\alpha_{\mu}}{2}} S_{(1)}^{\mu}}{\tilde{\lambda}_{\mu \mathrm{UE}}^{\frac{\alpha_{\mu}}{2}} I_{(1)}^{\mu}+\sigma^{2}} .
$$

Based on (A.1), the throughput $\mathrm{C}_{\mu}$ can be derived by using [40, Lemma 1], which is as follows

$$
\begin{aligned}
\mathrm{C}_{\mu} & =\frac{(1-\tau) T}{T} \mathrm{BW}_{\mu} \mathbb{E}\left[\log _{2}\left(1+\frac{\kappa_{\mu \mathrm{UE}}^{\frac{\alpha_{\mu}}{2}} S_{(1)}^{\mu}}{I_{(1)}^{\mu}+\widetilde{\lambda}_{\mu \mathrm{UE}}^{\frac{-\alpha_{\mu}}{2}} \sigma^{2}}\right)\right] \\
& =\frac{1-\tau}{\ln 2} \mathrm{BW}_{\mu} \int_{0}^{\infty} \frac{\varphi_{\mu}(t)}{t} e^{-\widetilde{\lambda}_{\mu \mathrm{UE}}^{\frac{-\alpha_{\mu}}{2}} \sigma^{2} t} d t
\end{aligned}
$$

where $\varphi_{\mu}(t)$ is

$$
\begin{aligned}
\varphi_{\mu}(t) & =\mathbb{E}\left[\left(1-e^{-t \kappa_{\mu \mathrm{UE}}^{\frac{\alpha_{\mu}}{2}} S_{(1)}^{\mu}}\right) e^{-t I_{(1)}^{\mu}}\right] \\
& \approx \int_{r_{o}}^{\infty}\left(1-e^{-t \kappa_{\mu \mathrm{UE}}^{\frac{\alpha_{\mu}}{2}} N P_{\mathrm{UE}_{o}}^{\mu} L(r)}\right) \underbrace{\mathbb{E}\left[e^{\left.-t I_{(1)}^{\mu}\right]} \widetilde{f}_{|X|}(r) d r .\right.}_{\widetilde{I}_{\mu}(t)}
\end{aligned}
$$

In (A.3), $\left\|\mathbf{h}_{o}\right\|^{2} \approx N$ with large $N, r_{o}$ is the reference distance to avoid singularity at zero, and $\widetilde{f}_{|X|}(\cdot)$ is the modified PDF of the distance $|X|$ between a $\mu \mathrm{UE}$ and its nearest sub- $6 \mathrm{GHz}$ BS under the condition $|X| \geq r_{o}$, which is

$$
\widetilde{f}_{|X|}(r)=\frac{2 \pi r}{e^{-\pi r_{o}^{2}}} \exp \left(-\pi r^{2}\right), \quad r \geq r_{o} .
$$

Note that the harvested ambient RF energy is much smaller than the directed transferred energy [2], [23], [24] and can be negligible, which is also illustrated in the simulation results of Section V in this paper. Therefore, based on the instantaneous harvested power given by (1), $P_{\mathrm{UE}_{o}}^{\mu}$ can be evaluated as

$$
P_{\mathrm{UE}_{o}}^{\mu} \stackrel{(a)}{\approx} \hbar_{\mu} L(r)
$$

where $\hbar_{\mu}=\frac{\tau}{1-\tau} \eta_{\mu} P_{\mu} N \lambda_{\mu}^{\frac{\alpha_{\mu}}{2}}$, step (a) is obtained from the Mapping theorem. Since the minimum distance between the typical BS and the interfering UEs is small in dense networks [41], by using the Laplace functional of PPP [37], $\widetilde{I}_{\mu}(t)$ is given by

$$
\widetilde{I}_{\mu}(t)=\exp \left(-2 \pi \int_{r_{o}}^{\infty} \mathbb{E}\left[\frac{t P_{\mathrm{MD}_{i}}^{\mu} L(x)}{1+t P_{\mathrm{MD}_{i}}^{\mu} L(x)}\right] x d x\right) .
$$

According to (A.5), we have

$$
\begin{aligned}
& \mathbb{E}\left[\frac{t P_{\mathrm{MD}_{i}}^{\mu} L(x)}{1+t P_{\mathrm{MD}_{i}}^{\mu} L(x)}\right] \\
& \quad=\frac{2 \pi}{e^{-\pi r_{o}^{2}}} \int_{r_{o}}^{\infty} \frac{t \hbar_{\mu} L(y) L(x)}{1+t \hbar_{\mu} L(y) L(x)} y e^{-\pi y^{2}} d y .
\end{aligned}
$$

Substituting (A.7) into (A.6), we obtain $\widetilde{I}_{\mu}(t)$ as (44) and complete the proof.

\section{APPENDIX B}

\section{A Detailed Derivation of (45)}

In the dense cellular networks, the noise power is negligible. Thus, based on (A.1), $\mathrm{C}_{\mu}$ can be calculated as

$$
\mathrm{C}_{\mu} \stackrel{(b)}{\approx} \frac{(1-\tau) T}{T} \mathbb{E}\left[\log _{2}\left(1+\kappa_{\mu \mathrm{UE}}^{\frac{\alpha \mu}{2}} N \frac{\widetilde{S}_{(1)}^{\mu}}{I_{(1)}^{\mu}}\right)\right],
$$

where step (b) is obtained by considering $\left\|\mathbf{h}_{o}\right\|^{2} \approx N$ with large $N$, and $\widetilde{S}_{(1)}^{\mu}=P_{\mathrm{UE}_{o}}^{\mu} L\left(\left|X_{o}\right|\right)$.

By using Jensen's inequality [42], (B.1) can be lower bounded as

$$
\mathrm{C}_{\mu}^{\mathrm{L}}=(1-\tau) \log _{2}\left(1+\kappa_{\mu \mathrm{UE}}^{\frac{\alpha_{\mu}}{2}} N \frac{e^{\mathbb{E}\left[\ln \widetilde{S}_{(1)}^{\mu}\right]}}{\mathbb{E}\left[I_{(1)}^{\mu}\right]}\right) .
$$


Considering $P_{\mathrm{UE}_{o}}^{\mu} \approx \hbar_{1} L(r)$ given in (A.5), we first calculate $\mathbb{E}\left[\ln \widetilde{S}_{(1)}^{\mu}\right]^{o}$ as

$$
\begin{aligned}
\mathbb{E}\left[\ln \widetilde{S}_{(1)}^{\mu}\right] & =\mathbb{E}\left[\ln P_{\mathrm{UE}_{o}}^{\mu}\right]+\mathbb{E}[\ln L(r)] \\
& =\ln \hbar_{1}+2 \mathbb{E}[\ln L(r)],
\end{aligned}
$$

where $\mathbb{E}[\ln L(r)]$ is

$$
\begin{aligned}
\mathbb{E} & {[\ln L(r)] } \\
& =\ln \beta_{\mu}-\alpha_{\mu} \int_{r_{o}}^{\infty} \ln (r) \widetilde{f}_{|X|}(r) d r \\
& =\ln \beta_{\mu}+\frac{\alpha_{\mu}}{2} e^{\pi r_{o}^{2}}\left(E i\left(-r_{o}^{2} \pi\right)-2 e^{-r_{o}^{2} \pi} \ln r_{o}\right) .
\end{aligned}
$$

In (B.4), $\widetilde{f}_{|X|}(\cdot)$ is given by (A.4).

With the help of the Campbell's theorem, $\mathbb{E}\left[I_{(1)}^{\mu}\right]$ in (B.2) is calculated as

$$
\begin{aligned}
\mathbb{E}\left[I_{(1)}^{\mu}\right] & =\mathbb{E}\left[P_{\mathrm{UE}_{i}}^{\mu}\right] 2 \pi \beta_{\mu} \int_{r_{o}}^{\infty} x^{1-\alpha_{\mu}} d x \\
& =\mathbb{E}\left[P_{\mathrm{UE}_{i}}^{\mu}\right] 2 \pi \beta_{\mu} \frac{r_{o}^{2-\alpha_{\mu}}}{\alpha_{\mu}-2},
\end{aligned}
$$

where $\mathbb{E}\left[P_{\mathrm{UE}_{i}}^{\mu}\right]$ can be similarly obtained based on (A.5), which is

$$
\begin{aligned}
\mathbb{E}\left[P_{\mathrm{UE}_{i}}^{\mu}\right] & \approx \hbar_{1} \beta_{\mu} \int_{r_{o}}^{\infty} r^{-\alpha_{\mu}} \widetilde{f}_{|X|}(r) d r \\
& =\hbar_{1} \beta_{\mu} \pi e^{\pi r_{o}^{2}} r_{o}^{2-\alpha_{\mu}} E_{\left(\frac{\alpha_{\mu}}{2}\right)}\left(r_{o}^{2} \pi\right) .
\end{aligned}
$$

Substituting (B.3) and (B.5) into (B.2), we can obtain (45) and complete the proof.

\section{APPENDIX C \\ A Detailed Derivation of (47)}

Similar to (A.3), we have

$$
\begin{aligned}
\mathrm{C}_{\mathrm{mm}} & =\frac{(1-\tau) T}{T} \mathrm{BW}_{\mathrm{mm}} \mathbb{E}\left[\log _{2}\left(1+\operatorname{SINR}_{\mathrm{mm}}\right)\right] \\
& =\frac{1-\tau}{\ln 2} \mathrm{BW}_{\mathrm{mm}} \int_{0}^{\infty} \frac{\varphi_{\mathrm{mm}}(t)}{t} e^{-\sigma^{2} t} d t
\end{aligned}
$$

where $\varphi_{\mathrm{mm}}(t)$ is

$$
\begin{aligned}
\varphi_{\mathrm{mm}}(t)= & \mathbb{E}\left[\left(1-e^{-t S_{\left(\lambda_{\mathrm{mm}}\right)}^{\mathrm{mm}}}\right) e^{-t I_{\tilde{(}_{\mathrm{mmUE}}}^{\mathrm{mm}}}\right] \\
= & \int_{r_{o}}^{R_{\mathrm{LoS}}}\left(1-e^{-t P_{\mathrm{UE}_{o}}^{\mathrm{mm}_{0}} \mathrm{M}_{D} \mathrm{M}_{B} L(r)}\right) \\
& \times \underbrace{\mathbb{E}\left[e^{-t I_{\left(\tilde{\lambda}_{\mathrm{mmUE}}\right)}^{\mathrm{mm}}}\right]}_{\widetilde{I}_{\mathrm{mm}}(t)} \widetilde{f}_{|Y|}(r) d r,
\end{aligned}
$$

where $P_{\mathrm{UE}_{o}}^{\mathrm{mm}}=\hbar_{\mathrm{mm}} L(r)$, and $\widetilde{f}_{|Y|}(\cdot)$ is the modified PDF of the distance $|Y|$ between a mmUE and its nearest mmWave BS under the constraint $|Y| \geq r_{o}$, which is

$$
\widetilde{f}_{|Y|}(r)=\frac{2 \pi \lambda_{\mathrm{mm}} r}{e^{-\pi \lambda_{\mathrm{mm}} r_{o}^{2}}} \exp \left(-\pi \lambda_{\mathrm{mm}} r^{2}\right) .
$$

We next calculate $\widetilde{I}_{\mathrm{mm}}(t)$ as

$$
\begin{aligned}
& \widetilde{I}_{\mathrm{mm}}(t) \\
& =\exp \left\{-2 \pi \widetilde{\lambda}_{\mathrm{mmUE}}^{\mathrm{mm}} \int_{r_{o}}^{R_{\mathrm{LoS}}}\left(1-\mathbb{E}\left[e^{-t P_{\mathrm{UE}_{j}}^{\mathrm{mm}} \widetilde{G}_{j} L(y)}\right]\right) y d y\right\} .
\end{aligned}
$$

By using the law of total expectation, we can directly obtain

$$
\begin{aligned}
& \mathbb{E}\left[e^{-t P_{\mathrm{UE}_{j}}^{\mathrm{mm}} \widetilde{G}_{j} L(y)}\right] \\
& =\sum_{\ell \in\left\{\mathrm{M}_{\mathrm{B}}, \mathrm{m}_{\mathrm{B}}\right\}} \sum_{k \in\left\{\mathrm{M}_{\mathrm{D}}, \mathrm{m}_{\mathrm{D}}\right\}} \operatorname{Pr}_{\ell k} \mathbb{E}_{P_{\mathrm{UE}_{j}}^{\mathrm{mm}}}\left[e^{-t P_{\mathrm{UE}_{j}}^{\mathrm{mm}} \ell k L(y)}\right] \\
& =\sum_{\ell \in\left\{\mathrm{M}_{\mathrm{B}}, \mathrm{m}_{\mathrm{B}}\right\}} \sum_{k \in\left\{\mathrm{M}_{\mathrm{D}}, \mathrm{m}_{\mathrm{D}}\right\}} \operatorname{Pr}_{\ell k} \int_{r_{o}}^{R_{\mathrm{LoS}}} e^{-t \hbar_{\mathrm{mm}} L(z) \ell k L(y)} \widetilde{f}_{|Y|}(z) d z .
\end{aligned}
$$

Substituting (C.3) and (C.5) into (C.4), after some manipulations, we obtain (49), and complete the proof.

\section{APPENDIX D}

\section{A Detailed DeRiVATION OF (51)}

In the noise-limited scenario, the throughput is calculated as

$$
C_{\mathrm{mm}}^{\mathrm{n}}=(1-\tau) \mathrm{BW}_{\mathrm{mm}} \mathbb{E}\left[\log _{2}\left(1+\frac{S_{(\lambda \mathrm{mm})}^{\mathrm{mm}}}{\sigma^{2}}\right)\right] .
$$

Considering the convexity of $\log _{2}\left(1+a e^{x}\right)$ for $a>0$ and using Jensen's inequality, the above can be lower bounded as

$$
C_{\mathrm{mm}}^{\mathrm{L}}=(1-\tau) \mathrm{BW}_{\mathrm{mm}} \log _{2}\left(1+\frac{e \zeta_{3}}{\sigma^{2}}\right),
$$

where $\zeta_{3}=\mathbb{E}\left[\ln \left(S_{\left(\lambda_{\mathrm{mm}}\right)}^{\mathrm{mm}}\right)\right]$. We can obtain $\zeta_{3}$ as

$$
\begin{aligned}
& \zeta_{3} \\
& =\mathbb{E}\left[\ln \left(P_{\mathrm{UE}_{o}}^{\mathrm{mm}_{o}} \mathrm{M}_{D} \mathrm{M}_{B} L\left(\left|Y_{o}\right|\right) \mathbf{1}\left(\left|Y_{o}\right|<R_{\mathrm{LoS}}\right)\right)\right] \\
& =\mathbb{E}\left[\ln \left(\hbar_{\mathrm{mm}} \mathrm{M}_{D} \mathrm{M}_{B} L^{2}\left(\left|Y_{o}\right|\right) \mathbf{1}\left(\left|Y_{o}\right|<R_{\mathrm{LoS}}\right)\right)\right] \\
& =\underbrace{\int_{r_{o}}^{R_{\mathrm{LoS}}}\left(\ln \left(\hbar_{\mathrm{mm}} \mathrm{M}_{D} \mathrm{M}_{B}\right)+2\left(\ln \beta_{\mathrm{LoS}}^{\mathrm{mm}}-\alpha_{\mathrm{LoS}}^{\mathrm{mm}} \ln r\right)\right) \widetilde{f}_{|Y|}(r) d r}_{\widetilde{\varphi}_{\mathrm{mm}}\left(\lambda_{\mathrm{mm}}\right)},
\end{aligned}
$$

where $\widetilde{f}_{|Y|}(\cdot)$ is given by (C.3). From (D.3), we see that $\widetilde{\varphi}_{\mathrm{mm}}\left(\lambda_{\mathrm{mm}}\right)$ increases with $\lambda_{\mathrm{mm}}$ because when more BSs are deployed, a mmUE becomes closer to its associated BS, which reduces the pathloss. After some manipulations, we can obtain $\widetilde{\varphi}_{\mathrm{mm}}\left(\lambda_{\mathrm{mm}}\right)$ as (52) and complete the proof.

\section{REFERENCES}

[1] L. Wang and K. K. Wong, "Energy coverage in wireless powered sub$6 \mathrm{ghz}$ and millimeter wave dense cellular networks," in Proc. IEEE Int. Conf. Commun. (ICC), Jul. 2017, pp. 1-6.

[2] K. Huang and X. Zhou, "Cutting the last wires for mobile communications by microwave power transfer," IEEE Commun. Mag., vol. 53, no. 6, pp. 86-93, Jun. 2015.

[3] F. Boccardi, R. W. Heath, Jr., A. Lozano, T. L. Marzetta, and P. Popovski, "Five disruptive technology directions for 5G," IEEE Commun. Mag., vol. 52, no. 2, pp. 74-80, Feb. 2014.

[4] J. G. Andrews et al., "What will 5G be?" IEEE J. Sel. Areas Commun., vol. 32, no. 6, pp. 1065-1082, Jun. 2014. 
[5] D. Liu et al., "User association in 5G networks: A survey and an outlook," IEEE Commun. Surveys Tuts., vol. 18, no. 2, pp. 1018-1044, 2nd Quart., 2016.

[6] X. Ge, S. Tu, G. Mao, and C. X. Wang, "5g ultra-dense cellular networks," IEEE Trans. Wireless Commun., vol. 23, no. 1, pp. 72-79, Feb. 2016.

[7] R. Zhang and C. K. Ho, "MIMO broadcasting for simultaneous wireless information and power transfer," IEEE Trans. Wireless Commun., vol. 12, no. 5, pp. 1989-2001, May 2013.

[8] Y. L. Che, L. Duan, and R. Zhang, "Spatial throughput maximization of wireless powered communication networks," IEEE J. Sel. Areas Commun., vol. 33, no. 8, pp. 1534-1548, Aug. 2015.

[9] I. Flint, X. Lu, N. Privault, D. Niyato, and P. Wang, "Performance analysis of ambient RF energy harvesting with repulsive point process modeling," IEEE Trans. Wireless Commun., vol. 14, no. 10, pp. 5402-5416, Oct. 2015.

[10] Z. Ding, S. M. Perlaza, I. Esnaola, and H. V. Poor, "Power allocation strategies in energy harvesting wireless cooperative networks," IEEE Trans. Wireless Commun., vol. 13, no. 2, pp. 846-860, Feb. 2014.

[11] A. H. Sakr and E. Hossain, "Analysis of K-tier uplink cellular networks with ambient RF energy harvesting," IEEE J. Sel. Areas Commun., vol. 33, no. 10, pp. 2226-2238, Oct. 2015.

[12] A. H. Sakr and E. Hossain, "Cognitive and energy harvesting-based D2d communication in cellular networks: Stochastic geometry modeling and analysis," IEEE Trans. Commun., vol. 63, no. 5, pp. 1867-1880, May 2015.

[13] H. H. Yang, J. Lee, and T. Q. S. Quek, "Heterogeneous cellular network with energy harvesting-based D2D communication," IEEE Trans. Wireless Commun., vol. 15, no. 2, pp. 1406-1419, Feb. 2016.

[14] G. Yang, C. K. Ho, R. Zhang, and Y. L. Guan, "Throughput optimization for massive MIMO systems powered by wireless energy transfer," IEEE J. Sel. Areas Commun., vol. 33, no. 8, pp. 1640-1650, Aug. 2015.

[15] F. Yuan, S. Jin, Y. Huang, K.-K. Wong, Q. T. Zhang, and H. Zhu, "Joint wireless information and energy transfer in massive distributed antenna systems," IEEE Commun. Mag., vol. 53, no. 6, pp. 109-116, Jun. 2015.

[16] Y. Zhu, L. Wang, K.-K. Wong, S. Jin, and Z. Zheng, "Wireless power transfer in massive MIMO aided HetNets with user association," IEEE Trans. Commun., vol. 64, no. 10, pp. 4181-4195, Oct. 2016.

[17] T. S. Rappaport et al., "Millimeter wave mobile communications for 5G cellular: It will work!" IEEE Access, vol. 1, pp. 335-349, May 2013.

[18] T. Bai, A. Alkhateeb, and R. W. Heath, Jr., "Coverage and capacity of millimeter-wave cellular networks," IEEE Commun. Mag., vol. 52, no. 9, pp. 70-77, Sep. 2014

[19] T. Bai and R. W. Heath, Jr., "Coverage and rate analysis for millimeterwave cellular networks," IEEE Trans. Wireless Commun., vol. 14, no. 2, pp. 1100-1114, Feb. 2015.

[20] S. Singh, M. N. Kulkarni, A. Ghosh, and J. G. Andrews, "Tractable model for rate in self-backhauled millimeter wave cellular networks," IEEE J. Sel. Areas Commun., vol. 33, no. 10, pp. 2196-2211, Oct. 2015.

[21] S. Ladan, A. B. Guntupalli, and K. Wu, "A high-efficiency $24 \mathrm{GHz}$ rectenna development towards millimeter-wave energy harvesting and wireless power transmission," IEEE Trans. Circuits Syst., vol. 61, no. 12 , pp. 3358-3366, Dec. 2014.

[22] M. Nariman, F. Shirinfar, A. P. Toda, S. Pamarti, A. Rofougaran, and F. D. Flaviis, "A compact $60-\mathrm{GHz}$ wireless power transfer system," IEEE Trans. Microw. Theory Techn., vol. 64, no. 8, pp. 2664-2677, Aug. 2016.

[23] L. Wang, M. Elkashlan, R. W. Heath, Jr., M. D. Renzo, and K. K. Wong, "Millimeter wave power transfer and information transmission," in Proc. IEEE Global Commun. Conf. (GLOBECOM), Dec. 2015, pp. 1-6.

[24] T. A. Khan, A. Alkhateeb, and R. W. Heath, Jr., "Millimeter wave energy harvesting," IEEE Trans. Wireless Commun., vol. 15, no. 9, pp. 6048-6062, Sep. 2016.

[25] E. Björnson, L. Sanguinetti, and M. Kountouris, "Deploying dense networks for maximal energy efficiency: Small cells meet massive MIMO," IEEE J. Sel. Areas Commun., vol. 34, no. 4, pp. 832-847, Apr. 2016.

[26] J. Park, S. L. Kim, and J. Zander, "Tractable resource management with uplink decoupled millimeter-wave overlay in ultra-dense cellular networks," IEEE Trans. Wireless Commun., vol. 15, no. 6 , pp. 4362-4379, Jun. 2016.

[27] H. Q. Ngo, E. G. Larsson, and T. L. Marzetta, "Energy and spectral efficiency of very large multiuser MIMO systems," IEEE Trans. Commun., vol. 61, no. 4, pp. 1436-1449, Apr. 2013.

[28] O. El Ayach, S. Rajagopal, S. Abu-Surra, Z. Pi, and R. W. Heath, Jr., "Spatially sparse precoding in millimeter wave MIMO systems," IEEE Trans. Wireless Commun., vol. 13, no. 3, pp. 1499-1513, Mar. 2014.
[29] S. Rangan, T. S. Rappaport, and E. Erkip, "Millimeter-wave cellular wireless networks: Potentials and challenges," Proc. IEEE, vol. 102 no. 3, pp. 366-385, Mar. 2014.

[30] W. Feng, Y. Wang, N. Ge, J. Lu, and J. Zhang, "Virtual MIMO in multi-cell distributed antenna systems: Coordinated transmissions with large-scale CSIT,” IEEE J. Sel. Areas Commun., vol. 31, no. 10, pp. 2067-2081, Oct. 2013.

[31] A. M. Hunter, J. G. Andrews, and S. Weber, "Transmission capacity of ad hoc networks with spatial diversity," IEEE Trans. Wireless Commun., vol. 7, no. 12, pp. 5058-5071, Dec. 2008.

[32] X. Lu, P. Wang, D. Niyato, D. I. Kim, and Z. Han, "Wireless networks with RF energy harvesting: A contemporary survey," IEEE Commun. Surveys Tuts., vol. 17, no. 2, pp. 757-789, 2nd Quart., 2015.

[33] K. Huang and V. K. N. Lau, "Enabling wireless power transfer in cellular networks: Architecture, modeling and deployment," IEEE Trans. Wireless Commun., vol. 13, no. 2, pp. 902-912, Feb. 2014

[34] L. Wang, H. Q. Ngo, M. Elkashlan, T. Q. Duong, and K. K. Wong, "Massive MIMO in spectrum sharing networks: Achievable rate and power efficiency," IEEE Syst. J., vol. 11, no. 1, pp. 20-31, Mar. 2017.

[35] B. F. Baccelli, B. Blaszczyszyn, and P. Muhlethaler, "An Aloha protocol for multihop mobile wireless networks," IEEE Trans. Inf. Theory, vol. 52, no. 2, pp. 421-436, Feb. 2006

[36] I. S. Gradshteyn and I. M. Ryzhik, Table of Integrals, Series and Products, 7th ed. San Diego, CA, USA: Academic, 2007.

[37] F. Baccelli and B. Blaszczyszyn, Stochastic Geometry and Wireless Networks Volume I-Theory. Hanover, Germany: Now Publishers, 2009.

[38] S. Deng, M. K. Samimi, and T. S. Rappaport, "28 GHz and $73 \mathrm{GHz}$ millimeter-wave indoor propagation measurements and path loss models," in Proc. IEEE Int. Conf. Commun. Workshop (ICCW), Sep. 2015, pp. $1244-1250$

[39] T. S. Rappaport, E. Ben-Dor, J. N. Murdock, and Y. Qiao, "38 GHz and $60 \mathrm{GHz}$ angle-dependent propagation for cellular \& peer-to-peer wireless communications," in Proc. IEEE Int. Conf. Commun. (ICC), Aug. 2012, pp. 4568-4573.

[40] K. A. Hamdi, "Capacity of MRC on correlated Rician fading channels," IEEE Trans. Commun., vol. 56, no. 5, pp. 708-711, May 2008.

[41] X. Lin, J. G. Andrews, and A. Ghosh, "Spectrum sharing for deviceto-device communication in cellular networks," IEEE Trans. Wireless Commun., vol. 13, no. 12, pp. 6727-6740, Dec. 2014.

[42] N. Lee, F. Baccelli, and R. W. Heath, Jr., "Spectral efficiency scaling laws in dense random wireless networks with multiple receive antennas," IEEE Trans. Inf. Theory, vol. 62, no. 3, pp. 1344-1359, Mar. 2016.

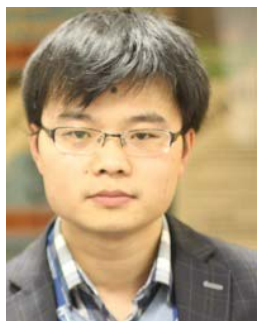

Lifeng Wang (M'16) received the Ph.D. degree in electronic engineering from the Queen Mary University of London in 2015. He is currently a Post-Doctoral Research Fellow with the Department of Electronic and Electrical Engineering, University College London. His research interests include millimeter-wave communications, massive MIMO, cloud-RAN, dense HetNets, ad hoc and sensor networks, caching and IoT-enabled networks, cognitive radio, physical layer security, and wireless energy harvesting. He received the Exemplary Editor Certificate of the IEEE COMMUNICATIONS LETTERS in 2016.

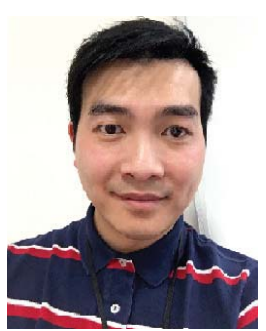

Kai-Kit Wong (M'01-SM'08-F'16) received the B.Eng., M.Phil., and Ph.D. degrees from The Hong Kong University of Science and Technology, Hong Kong, in 1996, 1998, and 2001, respectively, all in electrical and electronic engineering. $\mathrm{He}$ is currently a Professor of Wireless Communications with the Department of Electronic and Electrical Engineering, University College London, U.K. He is a fellow of the IET. He is a Senior Editor of the IEEE COMMUNiCATIONS LETTERS and the IEEE WIRELESS COMMUNICATIONS LETTERS. 


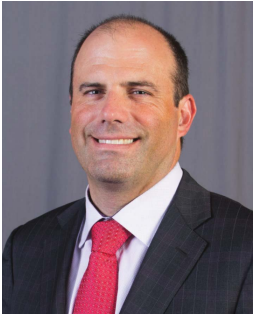

Robert W. Heath, Jr. (S'96-M'01-SM'06-F'11) received the B.S. and M.S. degrees from the University of Virginia, Charlottesville, VA, USA, in 1996 and 1997, respectively, and the Ph.D. degree from Stanford University, Stanford, CA, USA, in 2002, all in electrical engineering. From 1998 to 2001, he was a Senior Member of the Technical Staff and then a Senior Consultant with Iospan Wireless Inc., San Jose, CA, USA, where he was involved in the design and implementation of the physical and link layers of the first commercial MIMO-OFDM communication system. Since 2002, he has been with the Department of Electrical and Computer Engineering, The University of Texas at Austin, where he is currently a Cullen Trust for Higher Education Endowed Professor, and is a Member of the Wireless Networking and Communications Group. He is also the President and the CEO of MIMO Wireless Inc. He authored Introduction to Wireless Digital Communication (Prentice Hall, 2017), co-authored Millimeter Wave Wireless Communications (Prentice Hall, 2014), and authored Digital Wireless Communication: Physical Layer Exploration Lab Using the NI USRP (National Technology and Science Press, 2012).

Dr. Heath has been a Co-Author of 15 award winning conference and journal papers, including recently the 2010 and 2013 EURASIP Journal on Wireless Communications and Networking Best Paper Awards, the 2012 Signal Processing Magazine Best Paper Award, the 2013 Signal Processing Society Best Paper Award, the 2014 EURASIP Journal on Advances in Signal Processing Best Paper Award, the 2014 Journal of Communications and Networks Best Paper Award, the 2016 IEEE Communications Society Fred W. Ellersick Prize, and the 2016 IEEE Communications and Information Theory Societies Joint Paper Award. He was a Distinguished Lecturer in the IEEE Signal Processing Society. He is an ISI Highly Cited Researcher. He is also an elected member of the Board of Governors for the IEEE Signal Processing Society, a licensed Amateur Radio Operator, a Private Pilot, and a registered Professional Engineer in Texas.

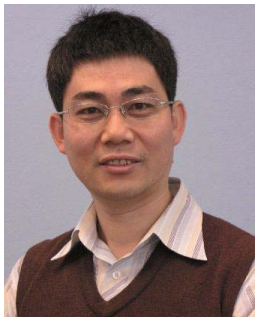

Jinhong Yuan (M'02-SM'11-F'16) received the B.E. and $\mathrm{Ph} . \mathrm{D}$. degrees in electronics engineering from the Beijing Institute of Technology, Beijing, China, in 1991 and 1997, respectively. From 1997 to 1999, he was a Research Fellow with the School of Electrical Engineering, The University of Sydney, Sydney, Australia. In 2000, he joined the School of Electrical Engineering and Telecommunications, University of New South Wales, Sydney, where he is currently a Telecommunications Professor. He is also a Co-Inventor of one patent on MIMO systems and two patents on low-density-parity-check codes. He has authored two books, three book chapters, over 200 papers in telecommunications journals and conference proceedings, and 40 industrial reports. His current research interests include error control coding and information theory, communication theory, and wireless communications. He has co-authored three Best Paper Awards and one Best Poster Award, including the Best Paper Award from the IEEE International Symposium on Wireless Communications Systems, Trondheim, Norway, in 2007, and the Best Paper Award from the IEEE Wireless Communications and Networking Conference, Cancun, Mexico, in 2011. He served as the IEEE NSW Chair of the Joint Communications/Signal Processions/Ocean Engineering Chapter from 2011 to 2014. $\mathrm{He}$ is currently serving as an Associate Editor of the IEEE TRANSACTIONS ON COMMUNICATIONS. 